\title{
Onion micelles with interpolyelectrolyte complex middle layer. Experimental motivation and computer study
}

Rahul Kumar Raya ${ }^{1}$, Miroslav Štěpánek ${ }^{1}$, Zuzana Limpouchová ${ }^{1}$, Karel Procházka ${ }^{1 *}$, Martin Svoboda $^{2,3}$, Martin Lísal ${ }^{2,3}$, Ewa Pavlova ${ }^{4}$, Athanasios Skandalis ${ }^{5}$ and Stergios Pispas ${ }^{5}$

${ }^{1}$ Department of Physical and Macromolecular Chemistry, Faculty of Science, Charles University in Prague, Hlavova 2030, 12840 Prague 2, Czech Republic

${ }^{2}$ Department of Physics, Faculty of Science, J. E. Purkinje University, České mládeže 8, 40096 Ústín. Lab., Czech Republic

${ }^{3}$ Department of Molecular and Mesoscopic Modelling, Institute of Chemical Process Fundamentals of the CAS, Rozvojová 135/1, 16502 Prague 6-Suchdol, Czech Republic ${ }^{4}$ Department of Polymer Morphology, Institute of Macromolecular Chemistry of the CAS, Heyrovský Square 2, 16000 Prague 6, Czech Republic

${ }^{5}$ Theoretical \& Physical Chemistry Institute, National Hellenic Research Foundation, 48 Vassileos Constantinou Ave., 11635 Athens, Greece

* To whom correspondence should be addressed

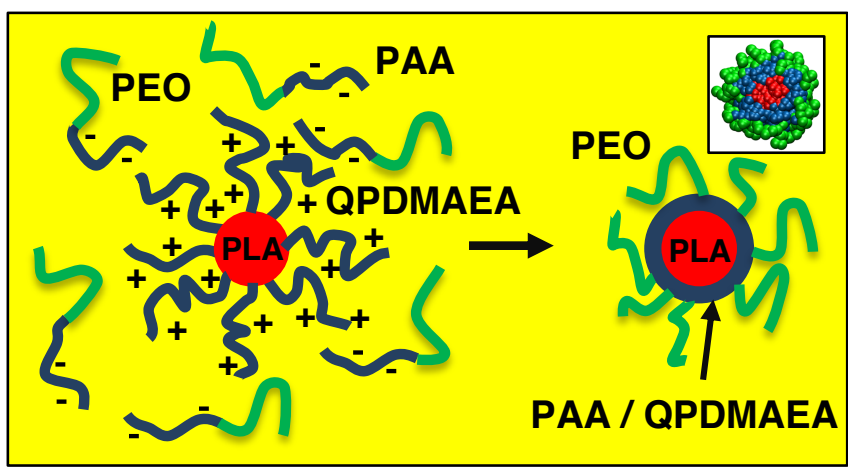

for Table of Contents use only 


\begin{abstract}
The paper describes successful preparation of onion micelles by sequential combination of self-assembly of amphiphilic polyelectrolytes (PE) in aqueous media and electrostatic coassembly of core/shell micelles formed in the first step with the oppositely charged doublehydrophilic copolymer. Experimental study and computer modeling (dissipative particle dynamics with explicit electrostatics) aimed at important features and trends of the assembly process were motivated by the controversy between the easiness of the process deduced from thermodynamic considerations and the low number of successful literature reports. The results show that onion micelles can be prepared if the studied system fulfils certain thermodynamic criteria and avoids kinetic obstacles. Nevertheless, the formation of well-developed onion micelles requires some hydrophobicity of PE backbones and their incompatibility with the soluble block. Otherwise, irregular low-density gel-like associates are formed. The increase in PE hydrophobicity and incompatibility promotes the assembly and improves the uniformity and spherical shape of onion micelles.
\end{abstract}

\title{
Key words
}

block polyelectrolytes, amphiphilic self-assembly, electrostatic co-assembly, onion micelles, light scattering, cryo-TEM microscopy, dissipative particle dynamics

\section{Introduction}

Amphiphilic block copolymers are theoretically interesting and application-attractive materials ${ }^{1-4}$ and consequently their behavior and properties have been extensively studied for several decades by experimentalists ${ }^{5-11}$ and theoreticians ${ }^{12-14}$. High-molar mass copolymers containing, e.g., polystyrene, poly(methyl methacrylate), or polyisoprene as a water-insoluble hydrophobic block, and e.g., poly(ethylene oxide), polyoxazoline or poly(methacrylic acid) as a water-soluble block are usually insoluble in aqueous media, but various long-term stable aqueous dispersions of their self-assembled nanoparticles can be prepared indirectly by their dissolution in aqueous mixtures with organic solvents and by (i) subsequent dialysis against aqueous buffers, (ii) selective evaporation of the organic solvent or (iii) by "quenching", which consists in fast injection of the copolymer dissolved in an organic solvent-rich mixture into an excess of the aqueous phase under vigorous stirring. ${ }^{7}, 15,16$ The simplest and the most 
frequently studied self-assembled nanoparticles are spherical micelles composed of compact insoluble cores and relatively expanded coronas (shells), but other structures, e.g., rod-like micelles, Janus micelles or vesicles have also been extensively studied. ${ }^{17-21}$ A decisive contribution to minimization of the Gibbs free energy accompanying the formation of copolymer micelles comes from the decrease in enthalpy due to the restriction of unfavorable interactions of insoluble blocks with solvent. As shown by a classical theoretical analysis three decades $\operatorname{ago}^{22-25}$, this contribution does not control the structural characteristics of the associates (association number, shape and inner structure). Entropy variations aiming at a maximum number of possible conformations of blocks in cores and in shells restricts the sizes of associates and plays the role of a stop-growth factor. Rigorously, the entropy-to-enthalpy interplay controls the association number, size and shape of associates and their inner structure, and the self-assembly can be classified as an enthalpy-driven and entropy-controlled process.

Another class of materials which offer promising applications in medicine, in various nanotechnologies and in a number of other fields ${ }^{26-31}$ comprises double-hydrophilic block copolymers composed of polyelectrolyte (PE) and neutral water-soluble blocks. Upon mixing two aqueous solutions of oppositely charged double-hydrophilic copolymers, or one doublehydrophilic copolymer with an oppositely charged homopolymer, electrostatic co-assembly takes place and stable dispersions of nanoparticles are formed with insoluble interpolyelectrolyte complex (IPEC) domains stabilized by the water-soluble blocks. ${ }^{32-37}$ Electrostatic co-assembly requires a number of opposite charges on co-assembling polyelectrolyte chains, but the electrostatic forces do not act as driving forces in the coassembly process. The electrostatic force between two charges kept in a certain distance from each other does not depend on whether the charged species are counterions or pendant groups on the PE chain and hence the change in the electrostatic interaction energy due to coassembly is small. Even though the cooperative effect of multiple interactions between oppositely charged PE chains promotes the association process, the decisive driving force is derived from a considerable increase in entropy. ${ }^{38}$ The overall entropy increases upon the formation of IPEC domains thanks to the release of mobile counterions into the bulk solvent. Interestingly, the association number, size, shape and the inner structure of co-assembled associates are not controlled either by electrostatics or by the entropy of the counterions, but depend on a number of other factors, mainly on the hydrophobicity/hydrophilicity of the PE 
blocks, and on their compatibility with neutral water-soluble blocks, on their lengths, flexibility and on interactions with small ions. ${ }^{39-43}$

A simultaneous or sequential application of several self- and/or co-assembling steps discussed above offers interesting possibilities for the preparation of multi-compartment associates. Several complex systems based on the combination of both processes, leading to multi-compartment micelles or to micelles with mixed corona have been already studied. ${ }^{44-53}$ This paper focuses on one specific type of complex nanoparticle, namely on multilayer onion micelles consisting of three (or more) chemically different layers: insoluble inner core, insoluble middle layer/layers and a stabilizing soluble shell at the periphery of the nanoparticle. The three-layer micelles can be prepared by the amphiphilic self-assembly in systems of $\mathrm{ABC}$ copolymers ${ }^{54-57}$, or in mixtures of $\mathrm{AB}$ and $\mathrm{BC}$ copolymers ${ }^{46,48-51,53}$, and by the electrostatic co-assembly of $\mathrm{AB}^{+}$and $\mathrm{C}^{-} \mathrm{D}$ copolymers, where $\mathrm{A}, \mathrm{B}, \mathrm{C}$ and $\mathrm{D}$ represent neutral blocks differing in solvophobicity/solvophilicity and $\mathrm{B}^{+}$and $\mathrm{C}^{-}$represent the oppositely charged PE blocks. A slightly simpler variant of the last method consists in mixing $\mathrm{AB}^{+}$micelles with long $\mathrm{C}^{-}$chains differing in length or at charge ratios of negative-to-positive charges appreciably differing from one. In spite of vast numbers of studies on electrostatic polyelectrolyte co-assembly, to the best of our knowledge, the last two variants have been only rarely exploited (and only the variant of $\mathrm{AB}^{+}$micelles with long $\mathrm{C}^{-}$chains). ${ }^{47,}{ }^{58-62}$ Therefore, we decided to investigate this process both experimentally and by computer simulations.

In the experimental part, we studied the co-assembly of core-shell micelles formed of poly(lauryl acrylate- $b$-quaternized (2-dimethylamino)ethyl acrylate), PLA- $b$-QPDMAEA (modified by the attachment of a dodecyl group at the QPDMAEA free end) with a double hydrophilic copolymer poly(acrylic acid)-b-poly(ethylene oxide), PAA- $b$-PEO by a combination of several experimental methods and we then performed extensive computer modeling in the second part. As the results of systematic experimental studies of the formation and properties of onion micelles (SLS, DLS, ITC, SANS, 2D NMR, etc.) for the fairly similar system of PLA- $b$-QPDMAEA-C12 with a slightly more hydrophobic PMA will be published in a separate paper, here we describe only a brief outline of scattering and microscopy results which clearly demonstrate the formation of onion micelles. The experimental part thus serves as a motivation for an extensive simulation study aimed at proving the proposed association scheme. 


\section{Experimental Section}

\section{Materials and Experimental Techniques}

\section{Polymers, chemicals, buffers}

Deionized water, $0.01 \mathrm{M}-0.05 \mathrm{M}$ aqueous solutions of $\mathrm{NaCl}$ (Aldrich), 0.01M aqueous solution of sodium tetraborate (Aldrich) and an aqueous mixture of $0.05 \mathrm{M} 2$ (cyclohehylamino) ethanesulfonic acid, CHES) (Aldrich) and $0.05 \mathrm{M} \mathrm{LiOH}$ (Aldrich) were used as buffers with $\mathrm{pH}$ ca 9.2 for preparation and studies of parent and onion micelles. Poly(acrylic acid)- $b$-poly(ethylene oxide), $\mathrm{PAA}_{104}-\mathrm{PEO}_{511}$, dispersity index, $D=1.3$ was purchased from Polymer Source, Inc., Dorval, Canada.

Cationic amphiphilic diblock poly(lauryl acrylate- $b$-quaternized (2-dimethylamino)ethyl acrylate) copolymer, $\mathrm{PLA}_{26}-b-\mathrm{QPDMAEA}{ }_{18}$, has been prepared by quaternization of the tertiary amine groups of the PDMAEA block of the PLA- $b$-PDMAEA diblock copolymer precursor with methyl iodide. The precursor amine diblock copolymer was synthesized by RAFT polymerization using AIBN as the radical initiator, 2-(dodecylthiocarbonothioylthio)2-methylpropionic acid as the chain transfer agent and 1,4-dioxane as the polymerization solvent. The chemical structure of PLA- $b$-QPDMAEA diblock copolymer is presented in scheme 1. Details on the synthesis and the characterization of the PLA- $b$-QPDMAEA ${ }^{63}$ are described in the Supporting Information

Scheme I. Chemical Structure of the PLA-b-QPDMAEA Diblock Copolymer.

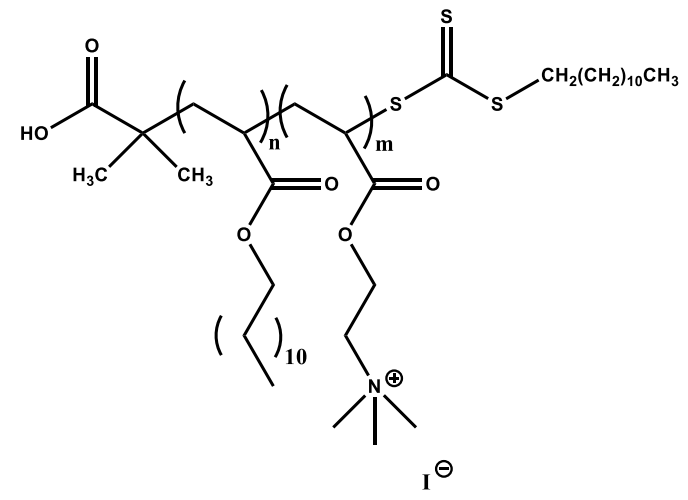




\section{Light scattering.}

The light scattering setup (ALV, Langen, Germany) consisted of a $22 \mathrm{~mW}$ He-Ne laser, operating at the wavelength $\lambda=632.8 \mathrm{~nm}$, an ALV CGS/8F goniometer, an ALV High QE APD detector and an ALV 5000/EPP multibit, multitau autocorrelator. The measurements were carried out at $25^{\circ} \mathrm{C}$ for the scattering angles, $\theta$, ranging from $30^{\circ}$ to $150^{\circ}$, corresponding to the scattering vector magnitudes, $q=\left(4 \pi n_{0} / \lambda\right) \sin (\theta / 2)$ (here $n_{0}$ is the refractive index of the solvent), from 6 to $25 \mu \mathrm{m}^{-1}$. Dynamic light scattering (DLS) measurements were evaluated by fitting the measured normalized time autocorrelation function of the scattered light intensity, $g^{(2)}(t, q)$, related to the electric field autocorrelation function, $g^{(1)}(t, q)$, by the Siegert relation, $g^{(2)}(t, q)=1+\beta\left|g^{(1)}(t, q)\right|^{2}$. The data were fitted either (i) with the aid of the constrained regularization algorithm (CONTIN) which provides the distribution of relaxation times $\tau$, $A(\tau, q)$, as the inverse Laplace transform of $g^{(1)}(t, q)$ function, or (ii) using the cumulant method.

Electrophoretic light scattering $\zeta$-Potential measurements were carried out with a Nano-ZS Zetasizer (Malvern Instruments, U.K.). $\zeta$-Potential values were calculated from electrophoretic mobilities (average of three subsequent measurements, each of which consisted of 15 runs) using the Henry equation in the Smoluchowski approximation, $\mu=\varepsilon \zeta / \eta$, where $\mu$ is the electrophoretic mobility and $\varepsilon$ is the dielectric constant of the solvent.

\section{Cryogenic Transmission Electron Microscopy.}

Cryo-TEM micrographs were obtained with a Tecnai G2 Spirit Twin 12 transmission electron microscope (FEI, Czech Republic) equipped with a cryo-specimen holder (Gatan, CA, USA). Sample preparation consisted in dropping $4 \mu \mathrm{L}$ of the sample solution on a TEM grid covered with a holey carbon supporting film. Before the experiment, the film was rendered hydrophilic by glow discharge (Expanded Plasma Cleaner; Harrick Plasma, Ithaca, NY, USA). The excess of the solution was removed by blotting (blotting time $1 \mathrm{~s}$; Whatman no. 1 filter paper) and the grid was immediately plunged into liquid ethane at $-181^{\circ} \mathrm{C}$. The frozen sample was transferred into the microscope and observed at $-173{ }^{\circ} \mathrm{C}$ using bright field imaging at $120 \mathrm{kV}$. 


\section{Results and Discussion}

\section{Experimental Section}

Before we start discussing the experimental results, it is useful to roughly estimate the sizes of ideal parent and onion micelles. This can be done on the basis of simple geometrical considerations. The cationic copolymer starts with a hydrophilic $-\mathrm{COOH}$ group and continues with 26 PLA monomeric units and later with 18 QPDMAEA units. Each PLA unit bears a relatively long pendant group consisting of one ester group and a dodecyl chain. This means that a 52-carbon long backbone bears 26 sidechains, each of them consisting of 14 atoms. The sidechains, which are only 3.7 times shorter than the whole block, are relatively crowded. Even though they are flexible, the PLA block behaves in non-polar media as a polymer brush $^{64}$ and adopts a fairly stretched, ca. 7 to $8 \mathrm{~nm}$ long conformation. In the studied system, PLA blocks are sterically restricted because they form dense micellar cores to prevent unfavorable interaction with water and are actually "tethered" to the core/shell interface. As the PLA chains have to fill a relatively large area close to the core-shell interface created by the connection of PLA and QPDMAEA blocks and moreover the carboxylic groups at the free end of the PLA block also tend to come close to the core-shell interface, their backbones adopt partially coiled conformations with flexible side chains arranged so as to fill the space and minimize the backbone stress. A simple estimate yields the maximum radius of the core $R_{\mathrm{C}}$ as ca. $8 \mathrm{~nm}$.

The QPDMAEA block is shorter than the PLA block and consists of 18 monomeric units, each bearing one elementary charge, i.e., its backbone is composed of 36 carbon atoms and every second $\mathrm{C}$ bears a four-atom-long linker terminated by a bulky and electrically charged trimethylamonium group. The QPDMAEA chains in micellar shells are expected to be stretched (up to $6 \mathrm{~nm}$ length) which means that the middle and periphery parts of the shell are relatively dilute. In the case of totally dehydrated cores, we estimated the geometrical radius of micelles as ca $15 \mathrm{~nm}$.

The PAA- $b$-PEO copolymer contains 104 PAA and 511 PEO units. The formation of ideal stoichiometric onion micelles with neutralized charges thus requires 5.8 fewer PAA chains than QPDMAEA chains in the shells of the parent micelles. This means that the "tethering density" of the PEO chains is low, but the number of PEO units stabilizing the onion micelles is high. The PEO blocks are long, flexible and stretched much less than the PE blocks. The ratio of PEO shell-forming units to PLA units in ideal stoichiometric micelles is 
ca. 3.4, and their ratio to the sum of PLA and IPEC-engaged PE units is ca. 1.5. It turns out that the onion micelles are, most probably, better stabilized by soluble blocks than the original parent micelles. Note that the ratio of soluble PE units in the shell of the original parent micelles to insoluble PLA units in the core is only ca. 0.7. Even though the stabilization of the parent micelles by the ionized shell is efficient, the QPDMAEA backbone is relatively short and quite hydrophobic, which restricts the solubility of the original micelles.

As PE chains in the IPEC layer collapse, the middle layer is thin (1 - $2 \mathrm{~nm})$. Based on classical description of the minimization of the core surface area exposed to the solvent, ${ }^{65}$ we assume that, in spite of the low "grafting density" of PEO blocks, the average shell density is similar to that of other PEO stabilized micelles ${ }^{46,49,66}$ because some PEO chains recoil back towards the core or their inner "tethered" parts partially collapse, thus increasing the density of the inner shell, which is necessary to protect the IPEC layer against interaction with water. This consideration leads to the conclusion that the size of the onion micelles (with respect to the parent micelles with the same association number of PLA chains) does not need to increase appreciably. As the studied system is not kinetically frozen and the stabilization of the onions seems to be very good, a reorganization of chains and decrease in the association number upon formation of the onions cannot be precluded. It this case, the onion micelles could be even smaller than the parent micelles.

Thermodynamic analysis suggests that the co-assembly of parent amphiphilic coreshell micelles with PE blocks with oppositely charged double-hydrophilic (PE-neutral) diblock copolymer chains is a favorable process. As the number of ionic groups on the PE shell of parent micelles is high, the electrostatic interaction is strong. The cooperative effect of multiple charges on micellar shells and the massive release of counterions in the bulk solvent upon the formation of the IPEC middle layer generate a strong driving force towards co-assembly. A mean-field investigation performed so far confirms the above assumption. ${ }^{37}$ However, to the best of our knowledge, the number of experimental studies that describe successful preparation of IPEC-stabilized onion micelles or related micellar associates based on amphiphilic core-shell micelles is surprisingly low ${ }^{44,47,59}$, which we think indicates severe experimental difficulties. The complications presumably ensue from the following facts. The formation of an insoluble IPEC layer requires not only suitable lengths and flexibility of oppositely charged PE blocks, but also their reasonable compatibility and some hydrophobicity of their (non-ionized) backbones as it was recently shown by computer simulations. $^{41,43}$ As most PEs are derived from fairly hydrophobic backbones (e.g., non- 
protonated poly(2-vinyl pyridine) is strongly hydrophobic) and the presence of pendant ionic groups generally increases the compatibility of different PEs, these thermodynamic requirements seem to be fulfilled. In our opinion, severe experimental obstacles follow from the high kinetic barrier of the process. In contrast to the spontaneous (sterically nonconstrained) formation of IPEC complexes from non-associated soluble components in bulk, or to the preparation of PE layer-by-layer films ${ }^{67-70}$, when the charged PE chains approach the oppositely charged PE layer without obstacles, the formation of onion micelles assumes the creation of a fairly thick insoluble IPEC layer (several $\mathrm{nm}$ ) and requires efficient penetration of PE blocks of the soluble component deep into the relatively dense shells of parent micelles. As soon as the PE blocks of the soluble component approach the oppositely charged PE shell, compact IPEC domains (insoluble in aqueous media) start to form at the shell periphery, which subsequently hinders further penetration of the PE chains into the inner shell. Our preliminary studies indicated that the formation of IPEC-stabilized onion micelles is relatively difficult. E.g., we did not succeed in preparing onion micelles based on parent micelles with kinetically frozen cores (polystyrene cores, unpublished data). Further, we observed that, in systems with dense shells, even the penetration of $\mathrm{C} 12$ surfactants is problematic and highly restricted. ${ }^{71}$ In the former case, rigid cores restrict the mobility of the shell-forming PE chains, which hinders the penetration of oppositely charged PE chains towards the core. In the latter case, the strong steric constraints in the inner shell prevent the formation of surfactant micelles and we found that, in contrast to common PE-surfactant systems, single surfactant molecules interact with PE chains.

In order to corroborate and exploit the concept of complex micelles with IPEC middle layers, which is so far not greatly supported by experimental data ${ }^{44,47,59}$, we performed an experimental study of the co-assembly in a mixture of copolymers for which we expected a surmountable kinetic barrier. To minimize problems with difficult diffusion of PE blocks of the second component into oppositely charged PE shells of pre-assembled micelles, we chose the strong polycation poly[lauryl acrylate- $b$-quaternized (2-dimethylamino)ethyl acrylate], PLA- $b$-QPDMAEA with a short PLA block and even shorter QPDMAEA as the first component. This copolymer is expected to form small micelles with expanded shells. The hydrophobic PLA block contains pendant lauryl sequences (very flexible) in each repeating unit. PLA has a very low glass-transition temperature, $T_{\mathrm{G}}$ ca. $-55^{0} \mathrm{C}{ }^{72}$, and the micelles with PLA cores formed by relatively short PLA chains, each of which starts with a hydrophilic carboxyl group, do not seem to be kinetically frozen. This is demonstrated by the fact that 
micelles form spontaneously upon dissolution of the sample in aqueous buffers and reorganize according to experimental conditions. Reversible association of the studied sample is a favorable condition for the study, but the PE block bears the C12 group from the chain transfer agent at its free end, which modifies and complicates the association behavior. We studied the behavior of hydrophobically modified chains in micellar shells some time ago ${ }^{66,73-}$ ${ }^{81}$ and found that a fraction of the modified shell-forming chains recoil back towards the core, but the other PE chains remain highly stretched with hydrophobic groups localized in the outermost part of the shell. At finite polymer concentrations, when the micelles are relatively close to each other, $\mathrm{C} 12$ groups behave as stickers and form bridges which temporarily interconnect several core/shell micelles. Another complication stems from the fact that the PE blocks are apt to hydrolyze. The hydrolysis is slow in neutral solutions around $\mathrm{pH} 7$, but accelerates in alkaline media, particularly at high $\mathrm{pH}$ values. During hydrolysis, the ester groups break and the shell-forming chains gradually lose their positively charged quaternary amino-groups and form negatively charged carboxylic groups, which increase the tendency of the core-shell micelles towards secondary aggregation. The experimental study described in the next part shows that the PLA- $b$-QPDMAEA-C12 solutions contain mixtures of small core/shell micelles with low fractions of fairly large micellar aggregates interconnected by hydrophobic bridges.

\section{Parent amphiphilic core-shell micelles with cationic PE shell}

Characterization of parent PLA-b-QPDMAEA-C12 micelles. Water and aqueous buffers are good solvents for the permanently charged QPDMAEA polyelectrolyte block and strong nonsolvents for the hydrophobic PLA block. As already mentioned, the copolymer contains a short (low $T_{\mathrm{G}}$ ) PLA block and a slightly shorter QPDMAEA block. The sample is watersoluble and the core-shell micelles form spontaneously upon its dissolution in aqueous media in a broad $\mathrm{pH}$ range. The formation of onion micelles in mixtures with PAA- $b$-PEO requires sufficient dissociation and ionization of the carboxylic groups in the PAA blocks, i.e., in the alkaline aqueous solutions. Because the ester groups in linkers attaching the quaternary nitrogen atoms can undergo hydrolysis at high $\mathrm{pHs}$, we studied and characterized the parent micelles in pure water and in moderately alkaline buffers with $\mathrm{pH}$ ca 9.2. Nevertheless, we found that the self-assembling behavior both in neutral and alkaline solutions is nonnegligibly affected by the presence of hydrophobic C12 groups and by electrostatic interactions. 
Light scattering measurements. The aqueous solutions of PLA- $b$-QPDMAEA-C12 micelles in water and in slightly alkaline media were first studied by wide angle static (SLS) and dynamic light scattering (DLS). Figure 1 shows the Berry plot of SLS measurements of parent micelles in $0.01 \mathrm{M}$ sodium tetraborate buffer. The angular dependences of the plotted data are strongly down-curved at low angles (low scattering vectors $q$ ) and do not yield realistic molar masses and radii of gyration of single core-shell micelles, but values that correspond to micellar aggregates. The measurements in pure water, salted water $(0.01,0.02$ and $0.05 \mathrm{M} \mathrm{NaCl})$ and in a 0.05 M CHES (2-(cyclohehylamino) ethanesulfonic acid)/Li buffer with $\mathrm{pH} 9.23$ provide similar down-curved shapes of both Berry and Zimm plots. All the results suggest that the presence of $\mathrm{C} 12$ sequences on the periphery on micellar shells induces some secondary aggregation of micelles. The extrapolation of data from the angular region 90 to $150^{\circ}$, where the scattering from large aggregates is suppressed, yields $R_{\mathrm{G}} \mathrm{ca} .60 \mathrm{~nm}$. The only differences between measurements in different buffers are the appreciably diverse values at low angles, which correspond to aggregates and depend strongly on the ionic strength of the solution. The lower the ionic strength, the lower are the extrapolated apparent molar masses and radii of gyration, which is understandable because the non-screened electrostatic repulsion hinders the secondary aggregation of the core-shell micelles. The average density of the associates based on extrapolated values from Figure $1\left(M_{\mathrm{w}}\right.$ ca. $8.3 \times 10^{6} \mathrm{~g} \cdot \mathrm{mol}^{-1}$ and $R_{\mathrm{G}}$ ca. $\left.150 \mathrm{~nm}\right)$ is unrealistically low $\rho$ ca. $10^{-2}$ g.cm $\mathrm{cm}^{-3}$ due to unwarranted extrapolation of wide-angle data through a broad angular region from $30^{\circ}$ to $0^{\circ}$. Moreover, the $z$-average $R_{\mathrm{G}}$ is affected more by large aggregates than the weight-average $M_{\mathrm{w}}$. Nevertheless, the extrapolated values indicate without any doubt that the micellar aggregates are very diffuse, low density (presumably gel-like) associates. Even though the classical wide-angle SLS measurements (treated as Zimm or Berry plots) do not provide information on single core-shell micelles, the SLS study and careful analysis of SLS data provide a useful overview on the self-assembling behavior. 


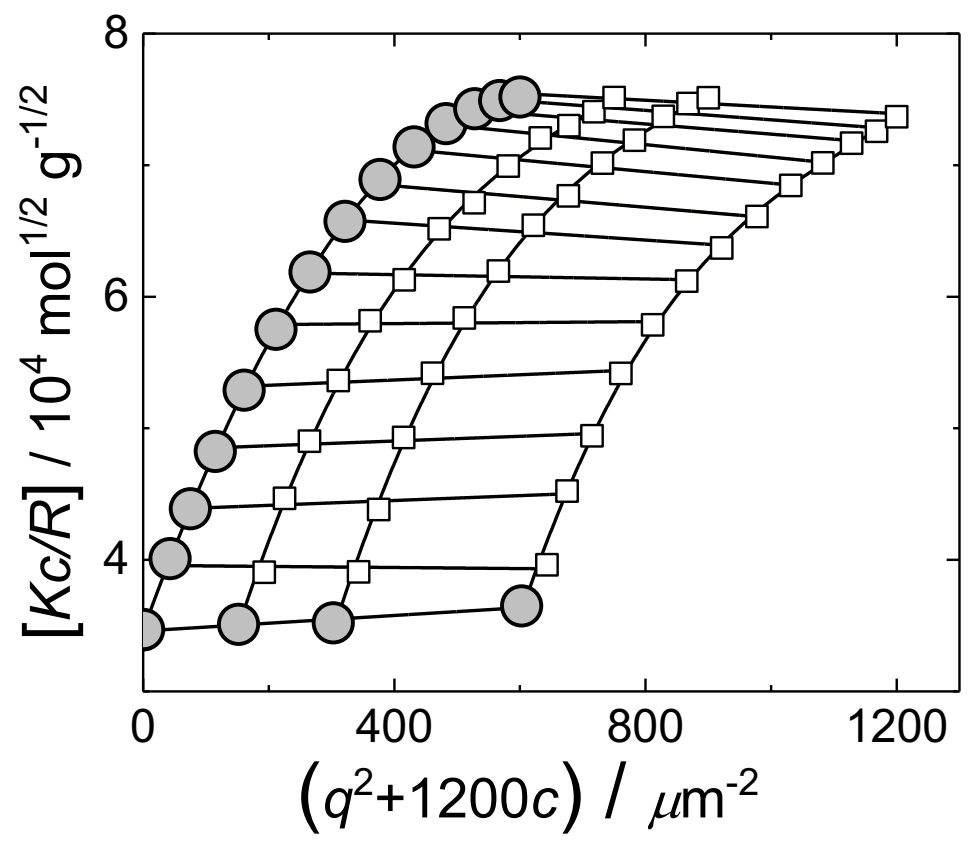

Figure 1. The Berry plot of parent PLA- $b$-QPDMAEA-C12 micelles in $0.01 \mathrm{M}$ sodium tetraborate buffer. The curves with squares (from the left to the right) correspond to polymer concentrations $0.125,0.25$ and $0.5 \mathrm{mg} / \mathrm{mL}$, respectively, and the curves with circles depict data extrapolated to the zero concentration and the zero angle.

Further information on the size distribution of the scattering particles was obtained by DLS. The distributions of the relaxation times are broad and the angular dependences of $z$ average $R_{\mathrm{H}}$ (evaluated by the method of cumulants developed by Koppel ${ }^{82}$ and recalculated from the diffusion coefficients under the assumption of unhindered diffusion of particles) do not yield constant values in the whole region of measured scattering angles $\left(30^{\circ}\right.$ to $\left.150^{\circ}\right)$. At large angles (high $q$ ), they are relatively constant (close to $55 \mathrm{~nm}$ ), but they strongly increase in the region of small angles (low q), reflecting the presence of large particles. The dependence of $R_{\mathrm{H}}$ on $q$ for a relatively low polymer concentration, $c=0.125 \mathrm{mg} / \mathrm{mL}$ in the $0.01 \mathrm{M}$ sodium tetraborate buffer is shown in Figure 2 as a typical example. 


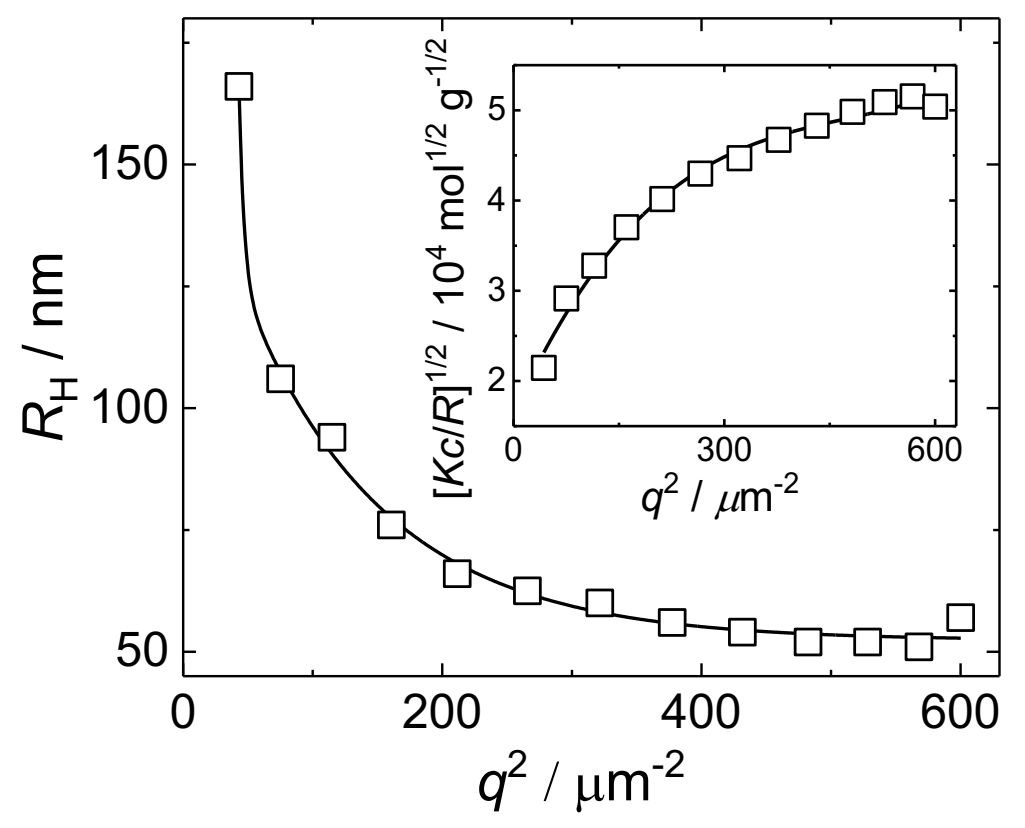

Figure 2. $R_{\mathrm{H}}$ of parent PLA- $b$-QPDMAEA-C12 micelles in $0.01 \mathrm{M}$ sodium tetraborate buffer recalculated from diffusion coefficients on the basis of the Stokes-Einstein formula as a function of $q^{2}$. Diffusion coefficients were obtained from the 2 nd order cumulant fit from DLS measurements of the solution with the concentration $0.125 \mathrm{mg} / \mathrm{mL}$ at the scattering angle $90^{\circ}$. Insert shows the corresponding SLS data.

It is interesting to compare the distributions of $R_{\mathrm{H}}$ for solutions with the same polymer concentration at low and high $q$. The comparison for the system in $0.1 \mathrm{M}$ sodium tetraborate is shown in Figure 3. While the distributions at low scattering angles are broad and show a pronounced tail towards high $R_{\mathrm{H}}$ caused by almost unattenuated scattering from large aggregates, a bimodal distribution is obtained at high $q$ (for the same damping constant). We assume that the relatively narrow peak with average $R_{\mathrm{H}}$ ca $30 \mathrm{~nm}$ corresponds to single coreshell micelles and the relatively broad peak spanning from 60 to $200 \mathrm{~nm}$ corresponds to micellar aggregates and, in the next part, we tried to corroborate this assumption by microscopy measurements. 


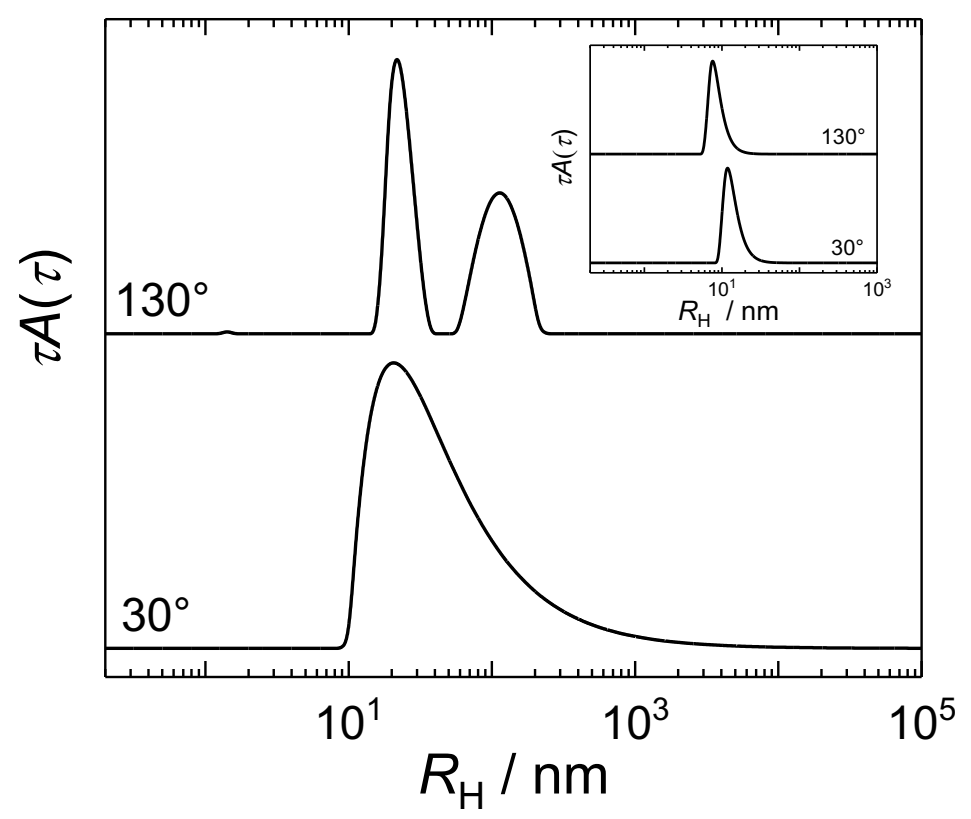

Figure 3. The intensity-weighted DLS distributions (and the number weighted DLS distributions shown in Insert) of hydrodynamic radii for parent PLA- $b$-QPDMAEA-C12 micelles with concentration $0.125 \mathrm{mg} / \mathrm{mL}$ in $0.01 \mathrm{M}$ sodium tetraborate buffer measured at scattering angles $130^{\circ}$ and $30^{\circ}$.

\section{Microscopy imaging}

The cryogenic transmission electron microscopy images (Cryo-TEM) of parent micelles are shown in Fig. 4a together with onion micelles (Fig 4 b, for their description and discussion see the later part). The side-by-side presentation of both objects was included for the comfort of readers because it facilitates the comparison of their sizes. The Cryo-TEM technique allows for direct imaging of nanoparticles in aqueous dispersions. The technique is based on rapid cooling (cooling rate of $\sim 10^{5} \mathrm{~K} / \mathrm{s}$ ) of a thin layer of dispersion embedded in holes in an amorphous carbon film by liquid ethane at a temperature of ca. $95 \mathrm{~K}$. Rapidly cooled water forms electron-transparent amorphous ice which enables observation of trapped nanoparticles under high vacuum conditions. However, it was reported that the imaged nanostructures can be segregated according to their size as those which are too large to be accommodated in the layer cannot be trapped. ${ }^{83}$ This explains why only small particles with diameters of ca. $30 \pm 4$ $\mathrm{nm}$ (mean \pm s.d.) were observed (Figure 4). Moreover, when comparing micelle size distributions obtained by Cryo-TEM and DLS, it is necessary to keep in mind that (i) the contribution from larger particles is overestimated in DLS intensity-weighted distributions 
even at high scattering angles and (ii) the outer hydrated layers of the micellar corona which already contribute to hydrodynamic size might not have sufficient contrast in vitreous ice to by visible by Cryo-TEM. The small size of spherical micelles agrees with a geometrical estimate based on the length of the core and shell-forming blocks of PLA- $b$-QPDMAEA copolymer under the assumption that the strongly hydrophobic core is densely filled by PLA blocks and the PE shell is relatively stretched.
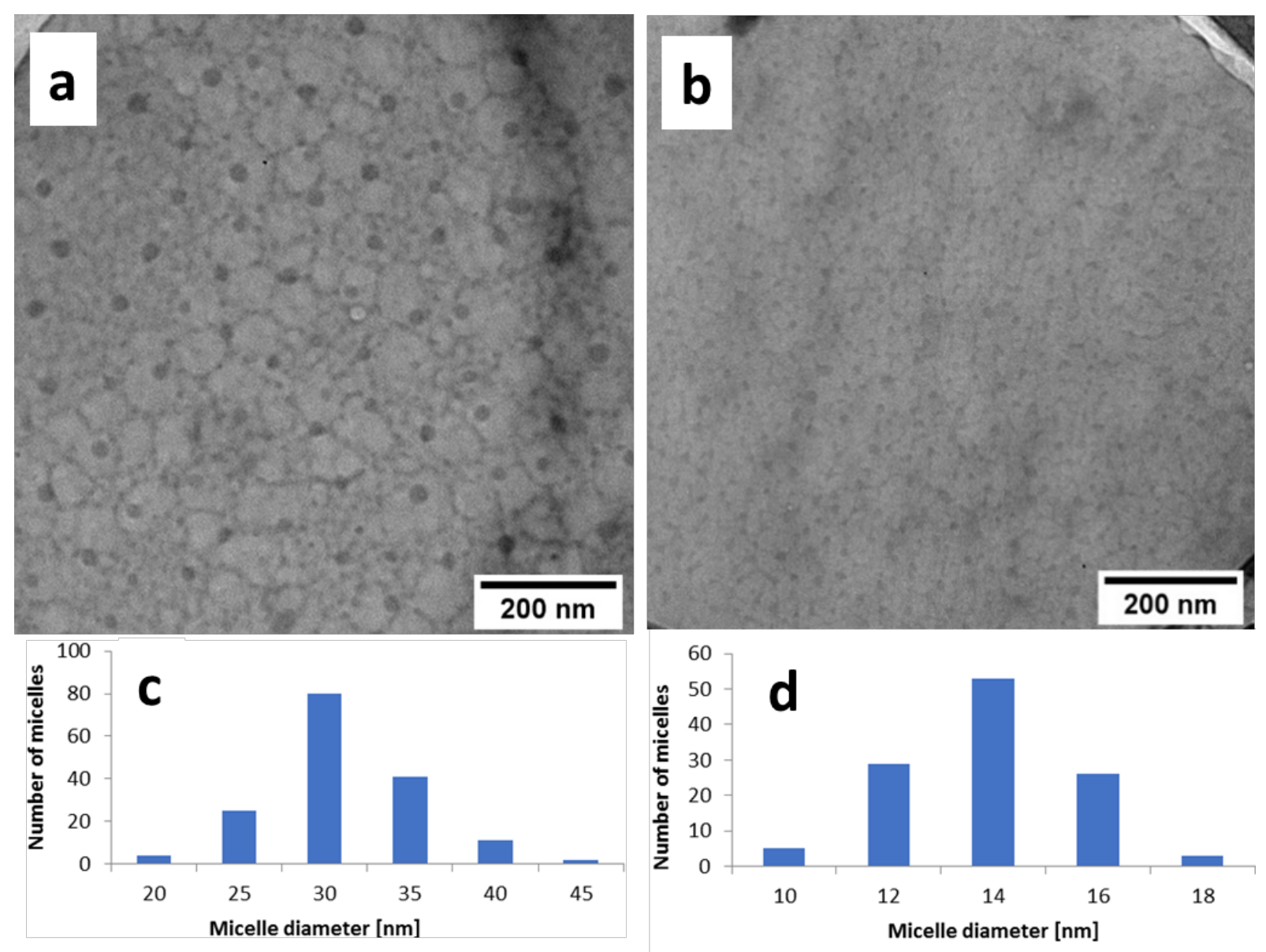

Figure 4. (a,b) Cryo-TEM images and (c,d) histograms of micelle diameters obtained from the analysis of the images of $(a, c)$ parent PLA-b-QPDMAEA micelles and $(b, d)$ onion micelles formed in a stoichiometric mixture of the components.

\section{Preparation and studies of onion micelles}

In the second part of the experimental study, we investigated the formation of onion micelles composed of inner PLA cores, mixed inter-polyelectrolyte QPDMAEA-PAA middle layers and stabilizing PEO shells in alkaline aqueous mixtures of parent PLA- $b$-QPDMAEA 
micelles with PAA- $b$-PEO solutions at different ratios, $Z$, of positive-to-negative charges on QPDMAEA and PAA chains. Because alkaline media are required for efficient ionization of PAA- $b$-PEO, but PLA- $b$-QPDMAEA-C12 undergoes slow hydrolysis at elevated $\mathrm{pH}$ values, we used moderately alkaline buffers with $\mathrm{pH} \cong 9$ and tried to minimize the time before mixing the components. The core/shell PLA- $b$-QPDMAEA-C12 micelles were first prepared in water. Then an aliquot of a relatively concentrated sodium tetraborate solution was added to achieve a copolymer concentration $0.5 \mathrm{mg} / \mathrm{mL}$ in $0.01 \mathrm{M}$ sodium tetraborate solution $(\mathrm{pH}$ 9.2). This solution was mixed under vigorous stirring with the PAA- $b$-PEO solution in $0.01 \mathrm{M}$ sodium tetraborate. A series of mixtures with different volume ratios (i.e., different charge ratios Z) were prepared. The stability of the onion micelles is expected to be better than that of the parent core/shell micelles because the chemically labile ester groups are embedded in insoluble IPEC domains and protected against the hydrolysis. The mixtures were left overnight with stirring and subsequently studied by scattering and microscopy techniques.

Based on the study of parent core/shell micelles, we cannot preclude that the solutions of onion micelles also contain the admixture of large aggregates and therefore the coassembly of core/shell micelles with the second copolymer was monitored by light scattering measurements at $90^{\circ}$. The assumption that onion micelles are accompanied by a low fraction of large micellar aggregates was checked and confirmed by LS measurements which provide the down-curved Berry plots and bimodal distributions of hydrodynamic radii (see the next part, Insert in Figure 6). The results of DLS and electrophoretic LS measurements are shown in Figure 5. Curve 1 shows the $\zeta$-potential vs. $Z$ measured by electrophoretic light scattering for mixtures of PLA- $b$-QPDMAEA parent micelles $(0.5 \mathrm{mg} / \mathrm{mL}$ in $0.01 \mathrm{M}$ sodium tetraborate) with increasing volumes of sodium tetraborate solutions of double-hydrophilic PAA- $b$-PEO copolymer. The $\zeta$-potential of pure parent micelles is positive (ca. $50 \mathrm{mV}$ ) and the curve decreases to negative values with increasing $Z$, which indicates the formation of complex nanoparticles with decreasing positive surface charge. According to expectations, the potential reaches $\zeta=0$ for $Z$ ca. 1, i.e., the measurement indicates the formation of strongly scattering nanoparticles with matched charges in the 1:1 inter-polyelectrolyte layer and zero charge at their outermost periphery. As $\zeta<0$ at $Z>1$, we can conclude that negatively overcharged particles form in the region of high $Z$. Even though the location of the Stern and shear layers in systems of polymeric micelles and the definition of the $\zeta$-potential are not as clear as in the case of compact colloidal particles, the measurement indicates gradual compensation of the fixed charge on micellar shells by the added copolymer and the build-up 
of an IPEC layer with mutually neutralized electric charges around the inner hydrophobic PLA core.

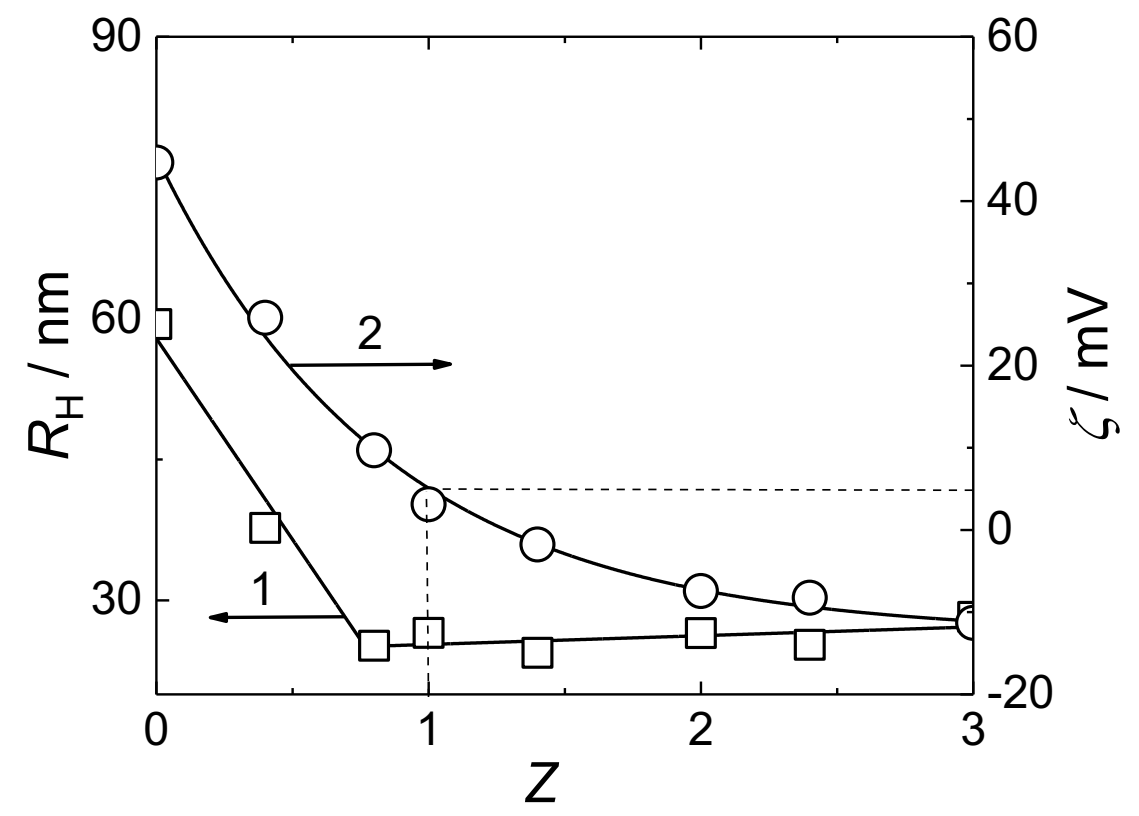

Figure 5. Hydrodynamic radii $R_{\mathrm{H}}$ (curve 1) and $\zeta$-potential (curve 2) of onion-shaped micelles formed in mixtures with constant concentration of the parent micelle-forming component, $c=0.5 \mathrm{mg} / \mathrm{mL}$ and increasing charge ratio, $Z$.

Curve 2 shows the $z$-average $R_{\mathrm{H}}$ vs. $Z$ curve. At first glance, the decrease in the $R_{\mathrm{H}}$ values with the increase in $Z$ may seem surprising, but this shape can be explained. When the onion micelles are formed, the stretched shell-forming QPDMAEA chains associate with the PAA chains, collapse and form a thin, compact IPEC layer. As already explained, the lengths of the cationic and anionic PE blocks differ appreciably and therefore the anchoring density of the PEO blocks is low and their number in the shells of the onion micelles is lower than in the parent micelles. In order to protect the IPAC layer against the aqueous medium, the inner parts of the PEO chains partially collapse and form a relatively dense inner layer decreasing the total thickness of the shell. ${ }^{46,49,66}$

For the reasons outlined above, we could not evaluate the molar masses of the onion micelles and therefore we tried to monitor the co-assembly process by measuring the intensity of light scattered from solutions with a constant concentration of the first component (parent core $/$ shell micelles, $c=0.5 \mathrm{mg} / \mathrm{mL}$ ) at $90^{\circ}$ vs. $Z$ (Figure 6). The monitoring provided valuable information and in combination with data shown in previous figure it enabled to draw a picture of the studied process. The SLS intensity slightly increases (ca. twice) with increasing 
$Z$ in the 0 to 1 region and for $Z>1$, it decreases. The increase in scattering intensity reflects the fact that the overall molar mass of onion micelles slightly increases. However, the increase is only moderate because the lengths of both PAA- $b$-PEO blocks are appreciably longer than those of PLA- $b$-QPDMAEA blocks and consequently the number of soluble units in onion micelles is higher than that in parent micelles. Because the 1:1 onion micelles are better stabilized than parent micelles (i.e., they contain higher ratio of soluble-to-insoluble units), the numbers of PLA- $b$-QPDMAEA chains, which participate at the formation the insoluble domains (central core and IPEC layer), decrease. As the inner core and the collapsed IPAC layer are very compact, i.e., relatively small, the overall size of onion micelles decreases.

In this paper, we did not study the structure and properties of non-stoichiometric micelles in detail and therefore we abstain from drawing definite conclusions concerning the decreasing part of the curve. Tentative explanation of the decrease at $Z>1$ can be outlined as follows. The Measurement of $\zeta$-potentials at high $Z$ indicate that the shells of onion micelles are slightly negatively overcharged. This means that only parts of PAA blocks participate in IPEC complex and other parts intermix with compatible PEO blocks and form a slightly ionized shell, analogously to onion micelles formed in mixtures of amphiphilic core-shell micelles with cationic shells and long anionic homopolyelectrolytes ${ }^{47}$ In short, the increase in the number of long negatively charged PAA chains leads to the formation a mixed and partly ionized PAA- $b$-PEO shell ${ }^{49}$ which increases the stability of dispersions. The increased electrostatic stabilization leads to the decrease in the number of PLA- $b$-QPDMAEA chains in the central core of onion micelles but the size remains approximately constant because the number of long PAA and PEO chains in the shell slightly increases and compensates the decrease in the number of PLA- $b$-QPDMAEA chains. The observed shape is consistent with experimental observations and simulation studies on simpler core/shell micelles with IPEC cores, which shows that the fractions and molar masses of co-assembled particles in nonstoichiometric mixtures with $Z \neq 1$ are lower than those in stoichiometric mixtures. ${ }^{36,41}$ In the studied system, the decrease in scattered light intensity for $Z>1$ is amplified, because the refractive index increment of onion micelles changes with $Z$ as the increment of the second component is lower than that of the first component, i.e., $(\mathrm{d} n / \mathrm{d} c)_{\text {PLA- } b \text {-QPDMAEA }}=0.230$ and $(\mathrm{d} n / \mathrm{d} c)_{\text {PAA- } b \text {-PEO }}=0.151 .{ }^{84}$ Relatively low negative $\zeta$-potentials at high $Z$, indicate that a major part of excess PAA- $b$-PEO chains remains dissolved in the solution in form of non-associate chains, analogously to electrostatically stabilized core/shell micelles ${ }^{43}$. 
The unambiguous interpretation of the observed dependence is almost impossible because the scattering intensity at constant finite angle and finite concentration reflects several contributions: (i) Molar mass of studied particles changes during the monitored process. (ii) As the system (particularly the parent micelles) are not kinetically frozen, the number of PLA- $b$-QPDMAEA chains in onions differ from that in parent micelles and in contrast to systems with kinetically frozen parent micelles which serve as growth centers, the concentration of scattering particles changes. (iii) Refractive increments of components differ. (iv) The scattering intensity at $90^{\circ}$ is affected by the change of the particle form factor as the size of the particles changes. (v) However, the main complications unreel from potential changes in the size and/or in the number of large aggregates which cannot be a priori precluded. Micellar aggregates dominate the scattering at finite angles in spite that their number fraction is negligible and their changes could affect the shape of the monitored curve considerably. Insert in Figure 6 shows the distribution of $R_{\mathrm{H}}$ in the system of stoichiometric onion micelles measured at $30^{\circ}$ and $130^{\circ}$ and at finite concentration, $c=0.5 \mathrm{mg} / \mathrm{mL}$. The distributions are bimodal and contain two well separated peaks (at the baseline level). The first peaks correspond to single onion micelles and the second peaks to micellar aggregates. At $130^{\circ}$ when the contribution of large particles is more suppressed by interference than at $30^{\circ}$, the first peak attains maximum at ca. $15 \mathrm{~nm}$, which shows that $R_{\mathrm{H}}$ of single onion micelles is smaller than that of parent micelles. This observation is consistent with other measurements. However, the size of aggregates also differs from that in system of parent micelles which suggests some changes in their size due to their interaction with PAA-b-PEO. This is why we did not attempt to interpret the whole shape of the SLS curve in detail.

The observation that the size of spherical onion micelles is smaller than that of the parent micelles was corroborated by cryo-TEM imaging. The sample for imaging was prepared as described in the part on parent micelles. The image (Figure $4 \mathrm{~b}$ ) shows a number of relatively uniform spherical particles. The diameter of spherical particles of ca. $14 \pm 2 \mathrm{~nm}$ (mean \pm s.d.) is unambiguously smaller than that of the parent core-shell micelles shown in Figure $4 b$. 


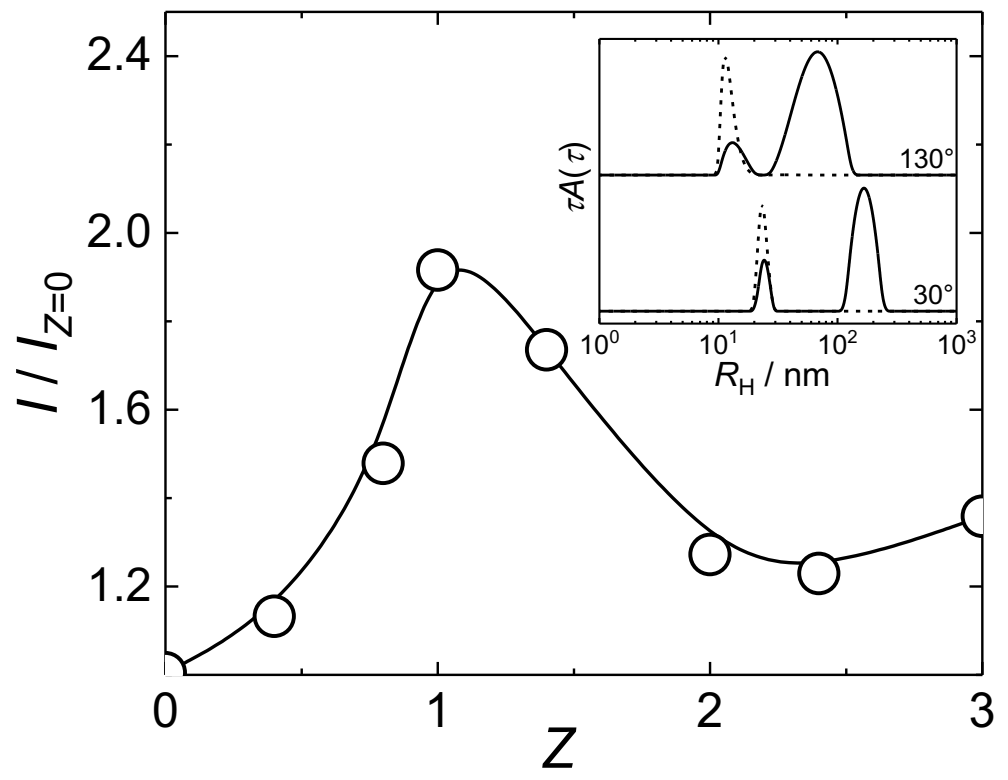

Figure 6. Normalized light scattering intensity of onion-shaped micelles formed in mixtures with increasing negative-to-positive charge ratio, $Z$, monitored at the scattering angle $90^{\circ}$. Insert: The intensity-weighted (solid line) and number-weighted (dashed line) DLS distributions of hydrodynamic radii of onion-shaped micelles at $Z=1$ and scattering angles $30^{\circ}$ and $130^{\circ}$.

\section{Computer Modelling Section}

To demonstrate that the co-assembling scheme proposed in the experimental part is sound from the thermodynamic point of view, we performed extensive computer modeling using Dissipative Particle Dynamics (DPD) as originally proposed by Hoogerbrugge and Koelman ${ }^{85}$ and later modified by Groot and Warren. ${ }^{86-88}$ DPD is a coarse-grained variant of molecular dynamics suitable for studying large complex systems, e.g., for complex polymer solutions and blends ${ }^{41,43,89-105}$. We explicitly included electrostatic interactions between charged DPD beads employing the Slater (exponential) charge smearing along with the relative permittivity of water $\varepsilon_{\mathrm{r}}=80 .{ }^{97}$ The principle of the DPD method together with all the simulation details are described in our recent papers $41,90,97,106$ and are also summarized in the Supporting Information. The choice of interaction parameters is elucidated below. It should be mentioned that the simulation study was not aimed at a faithful reproduction of the relatively complicated behavior of the experimentally studied system. The simulations are concerned with the general features and trends of complex co-assembly which combine amphiphilic and electrostatic mechanisms and leads to onion micelles with hydrophobic cores, IPEC middle layers and hydrophilic shells. 


\section{Simulation results}

We performed several series of simulations for systems differing in numbers of chains, in the length of blocks and in the amphiphilicity of the individual components. Interaction parameters used in simulations and their ranges are summarized in Table 1. Note that we present and discuss our results in terms of $\Delta a_{i j}=a_{i j}-\left(a_{i i}+a_{j j}\right) / 2$, which is proportional to the Flory-Huggins interaction parameter. Specification of individual systems and other pertinent details are given in Table 2.

Beads $\mathrm{A}$ are always strongly hydrophobic while beads $\mathrm{C}$ are hydrophilic and compatible with the counterions. Beads $\mathrm{B}^{+}$and $\mathrm{B}^{-}$are readily soluble in water because they bear electric charges $+1 e$ and $-1 e$, respectively. To investigate how the hydrophilicity/hydrophobicity of uncharged PE backbones and their compatibility with counterions influences the co-assembly and the structure of onion micelles, we varied interaction parameters $\Delta a_{\mathrm{BS}}, \Delta a_{\mathrm{BC}}$ and $\Delta a_{\mathrm{BI}}$ in a relatively broad range. The same variation of all three parameters minimizes the number of time-consuming simulations and can be supported by the following arguments: When the hydrophobicity of the PE backbone, $\Delta a_{\mathrm{BS}}$, increases, not only the solubility of charged PE blocks in water, but also their miscibility with polar water-soluble block $\mathrm{C}$ decrease, which is reflected in increasing $\Delta a_{\mathrm{BC}}$. Simultaneously, the interactions of the counterions with the PE blocks and particularly with IPEC worsen. The main restriction that hinders the penetration of ions into IPEC is of entropic origin and stems from the severely hindered motion of counterions in the dense IPEC domain. The second contribution is derived from the hydrophobicity of the IPEC domain with compensated charges. The non-associated PE blocks (non-engaged in IPEC formation) contain ionized groups and interact favorably with counterions both in solutions and also in the dry solid state, where they form polymeric salts, but the hydrophobic IPEC domain represents a fairly hostile medium for small ions. While the former effect is directly accounted for in DPD simulations with explicit electrostatics, the latter effect is modeled by changes in $\Delta a_{\mathrm{BI}}$.

To simplify the discussion: if we say that we study PEs differing in their hydrophobicity, this means that all three parameters, $\Delta a_{\mathrm{BS}}=\Delta a_{\mathrm{BI}}=\Delta a_{\mathrm{BC}}$, vary. We investigated mobile (not kinetically frozen) systems and therefore we began all the simulations from a random mixture of dispersed copolymer chains. However, we also performed a few simulations starting from the final configuration of a system with lower hydrophobicity to prove that the equilibrium state of the self-assembled system is independent of initial 
configurations. We used the reduced system density $\rho=3$ and employed $240 \mathrm{~A}_{5} \mathrm{~B}_{5}{ }^{+}$and 240 or $400 \mathrm{C}_{m} \mathrm{~B}_{n}^{-}$chains in cubic simulation boxes ranging from $32^{3}$ to $33.5^{3}$; see Table 2 . The systems were typically equilibrated depending on the hydrophobicity for a few $10^{6}$ time steps with the time step equal to 0.05 DPD reduced units. The time step in reduced units is defined as $\Delta t=\sqrt{\frac{k T}{m_{S} r_{c}^{2}}}$, where $m_{S}$ is the mass of a solvent bead. Data production runs spanned from $10 \cdot 10^{6}$ to $100 \cdot 10^{6}$ time steps depending on the hydrophobicity of the backbone.

\begin{tabular}{|c|c|c|c|c|}
\hline $\begin{array}{c}a_{i j} \\
\Delta a_{i j}=a_{i j}-a_{i i}\end{array}$ & A & $\mathrm{B}^{+}, \mathrm{B}^{-}$ & $\mathrm{C}$ & $\mathrm{S}, \mathrm{I}^{+}, \mathrm{I}^{-}$ \\
\hline A & $\begin{array}{c}25 \\
0\end{array}$ & $\begin{array}{l}40 \\
15\end{array}$ & $\begin{array}{l}40 \\
15\end{array}$ & $\begin{array}{l}40 \\
15\end{array}$ \\
\hline $\mathrm{B}^{+}, \mathrm{B}^{-}$ & & $\begin{array}{c}25 \\
0\end{array}$ & $\begin{array}{c}25-32.5 \\
0-7.5\end{array}$ & $\begin{array}{c}25-32.5 \\
0-7.5\end{array}$ \\
\hline $\mathrm{C}$ & & & $\begin{array}{c}25 \\
0\end{array}$ & $\begin{array}{c}25 \\
0\end{array}$ \\
\hline $\mathrm{S}, \mathrm{I}^{+}, \mathrm{I}^{-}$ & & & & $\begin{array}{c}25 \\
0\end{array}$ \\
\hline
\end{tabular}

Table 1: Interaction parameters $a_{i j}$ (the upper number) and their difference against the $a$ parameters describing interaction between like particles, $\Delta a_{i j}=a_{i j}-\left(a_{i i}+a_{j j}\right) / 2$ (the bottom number) which is proportional to the Flory-Huggins interaction parameter. Symbols A and C represent beads $\mathrm{A}$ and $\mathrm{C}$, respectively. $\mathrm{B}^{+}$and $\mathrm{B}^{-}$denote charged beads $\mathrm{B}$. $\mathrm{S}$ corresponds to the solvent and $\mathrm{I}^{+}$and $\mathrm{I}^{-}$to the positively and negatively charged counterions, respectively. 


\begin{tabular}{|c|c|c|c|}
\hline & SYSTEM I & SYSTEM II & SYSTEM III \\
\hline Composition & $240 \mathrm{~A}_{5} \mathrm{~B}_{5}^{+}$ & $240 \mathrm{~A}_{5} \mathrm{~B}_{5}^{+}$ & $240 \mathrm{~A}_{5} \mathrm{~B}_{5}^{+}$ \\
\hline Box size & $240 \mathrm{C}_{5} \mathrm{~B}_{5}^{-}$ & $240 \mathrm{C}_{7} \mathrm{~B}_{5}^{-}$ & $400 \mathrm{C}_{5} \mathrm{~B}_{3}^{-}$ \\
\hline \multirow{2}{*}{$\Delta a_{\mathrm{BS}}=\Delta a_{\mathrm{BC}}=\Delta a_{\mathrm{BI}}$} & $0 ; 2.5 ; 3.5 ; 5$ & $0 ; 2.5 ; 3.5 ; 5$ & 33.5 \\
& 32 & 33 & $0 ; 3.5 ; 5 ; 7.5$ \\
\hline$\Delta a_{\mathrm{BI}}$ & 0 & 0 & 0 \\
\hline
\end{tabular}

Table 2. Description of individual systems and their parameters.

\section{System I}

In the first series of simulations, we studied stoichiometric systems consisting of 240 amphiphilic copolymer chains $\mathrm{A}_{5} \mathrm{~B}_{5}^{+}$and 240 double-hydrophilic $\mathrm{C}_{5} \mathrm{~B}_{5}{ }^{-}$copolymer chains differing in the hydrophobicity of PE blocks $\mathrm{B}^{+}$and $\mathrm{B}^{-}$. The simulation results for a system with readily soluble PE backbones $\left(\Delta a_{\mathrm{BS}}=0\right)$ are depicted in Figure 7 . Figure 7 a compares the weight-average distributions of the association numbers of the parent and the corresponding onion micelles. The Insert in Figure 7a shows a typical simulation snapshot. A pure system of parent micelles formed by $\mathrm{A}_{5} \mathrm{~B}_{5}{ }^{+}$with $\Delta a_{\mathrm{AS}}=\Delta a_{\mathrm{AB}}=15$ and $\Delta a_{\mathrm{BS}}=\Delta a_{\mathrm{BC}}=0$ was studied in our recent paper. ${ }^{96}$ The study showed that the highly soluble copolymer with $\Delta a_{\mathrm{BS}}=0$ forms micelles, but the association number, $A_{\mathrm{S}}$, is low and the fraction of free unimers is quite high. The relatively narrow peak of the core/shell micelles, reproduced in Figure 7a, which spans from $A_{\mathrm{S}}=5$ to 15 and attains a maximum at $A_{\mathrm{S}}=7$, is only slightly separated from those of the unimers, dimers and trimers. The fraction of associates with $A_{\mathrm{S}}=7$ is only $66 \%$ of that of unimers, but the cumulative fraction of associates ranging from $A_{\mathrm{S}}=5$ to $15, \sum_{A_{S}=5}^{A_{S}=15} F_{w}\left(A_{S}\right)$, exceeds ca. 3.7 times that of the unimer. In our recent computer studies ${ }^{43}$, we found that the stoichiometric co-assembly of $\mathrm{C}_{n} \mathrm{~B}_{n}{ }^{+}$and $\mathrm{C}_{n} \mathrm{~B}_{n}{ }^{-}$, where $\mathrm{C}_{n}$ is the neutral water-soluble block and $\mathrm{B}_{n}{ }^{+}$and $\mathrm{B}_{n}{ }^{-}$are the PE blocks, leads to the formation of multichain associates only if the 
$\mathrm{PE}$ backbones are at least moderately hydrophobic, i.e., if $\Delta a_{\mathrm{BS}}>0$. If the $\mathrm{B}$ chains (without being charged) are water soluble, only dimers are formed because minimization of the number of interactions of $\mathrm{B}$ beads with water is not required. The formation of onion micelles is more complex than that of core/shell micelles and our present study shows that the electrostatic coassembly takes place in systems with $\Delta a_{\mathrm{BS}}=\Delta a_{\mathrm{BC}}=0$ (i.e., in systems with soluble PE chains which are compatible with $\mathrm{C}$ ). However, the association process is restricted and the association number of multichain associates is relatively low. The fractions of unimer and of small associates are important and the peak of larger associates is not fully separated from that of unimers. It attains a flat maximum between $A_{\mathrm{s}}=10$ and 13 . The fractions of associates close to the peak maximum are significantly lower than that of the unimer, but the peak of associates is relatively broad and comprises $A_{\mathrm{S}}$ from 7 to 27 and their cumulative fraction, $\sum_{A_{S}=7}^{A_{S}=27} F_{w}\left(A_{s}\right)$, exceeds that of the unimer ca. 2.8 times.

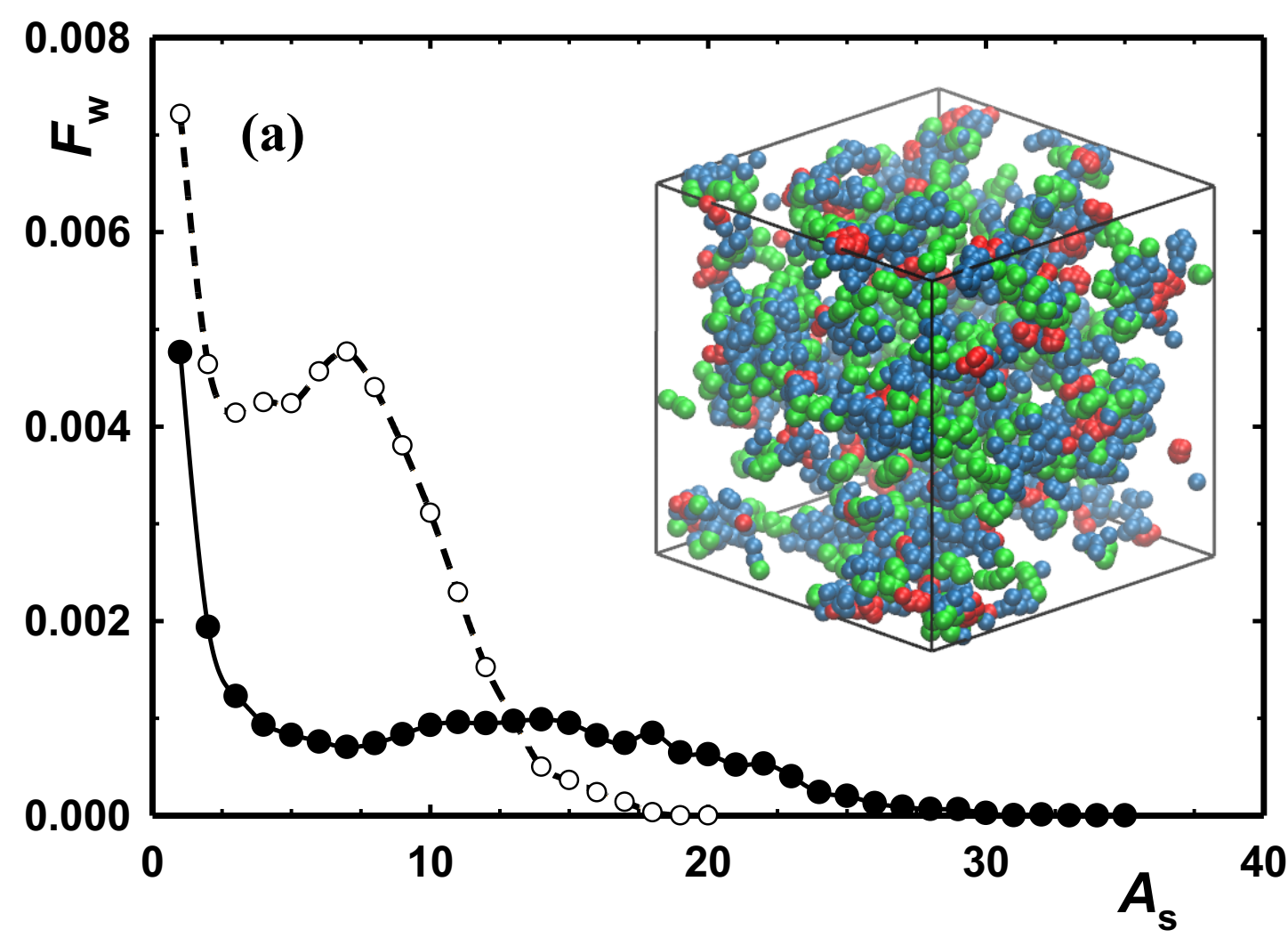




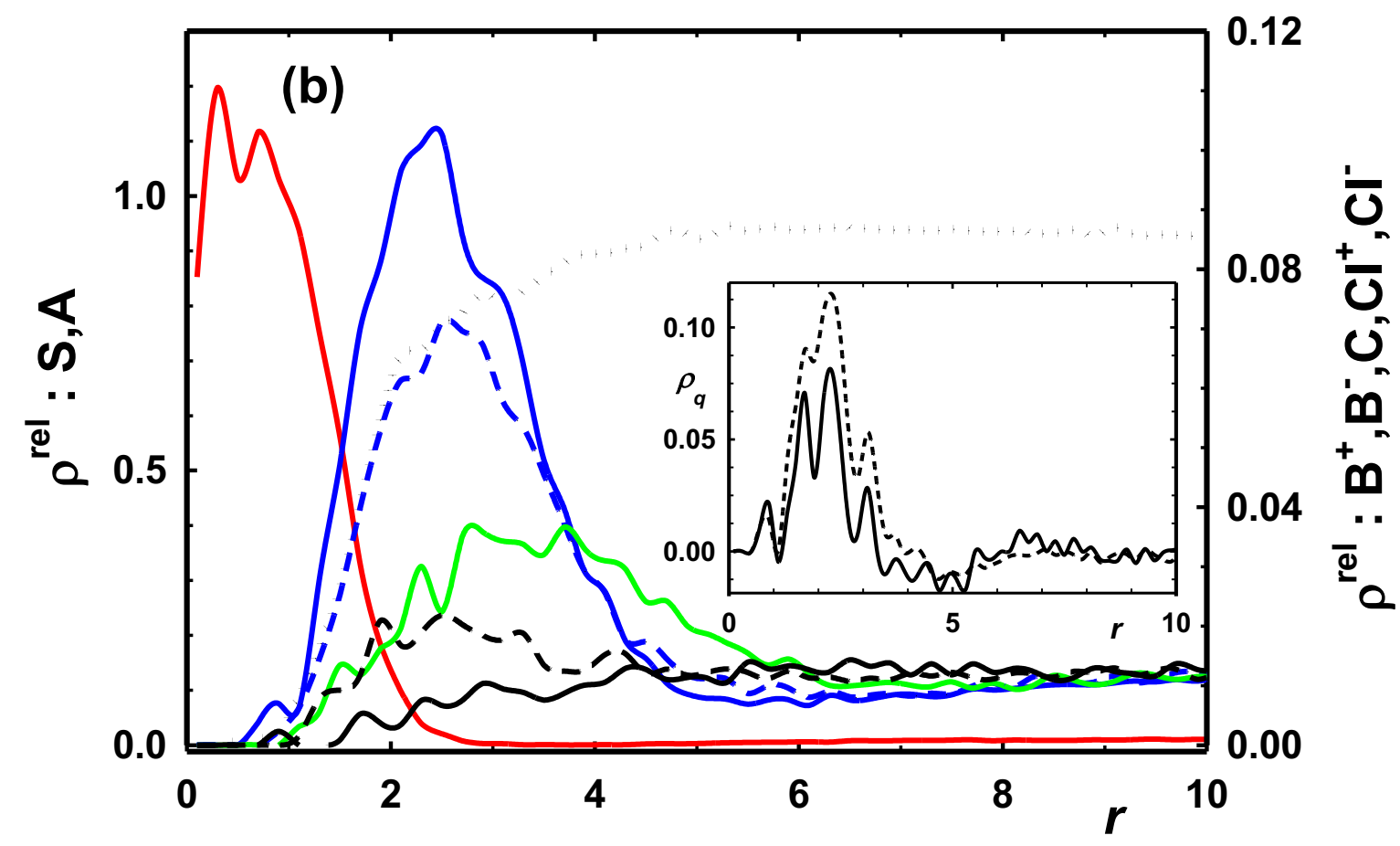

Figure 7. (a) Weight distributions functions, $F_{\mathrm{w}}\left(A_{\mathrm{s}}\right)$, of association numbers $A_{\mathrm{s}}$ in a system containing 240 copolymer chains $\mathrm{A}_{5} \mathrm{~B}_{5}{ }^{+}$(dashed curve with circles, parent system) and in the system containing 240 copolymer chains $\mathrm{A}_{5} \mathrm{~B}_{5}{ }^{+}$and 240 double-hydrophilic $\mathrm{C}_{5} \mathrm{~B}_{5}{ }^{-}$copolymer chains (solid curve with filled circles, System I) with parameters $\Delta a_{\mathrm{AS}}=\Delta a_{\mathrm{AB}}=\Delta a_{\mathrm{AI}}=\Delta a_{\mathrm{AC}}=15$ and $\Delta a_{\mathrm{BS}}=\Delta a_{\mathrm{BC}}=\Delta a_{\mathrm{BI}}=\Delta a_{\mathrm{Cl}}=0$. The insert in Figure 7a shows a typical snapshot for systems containing both $\mathrm{A}_{5} \mathrm{~B}_{5}{ }^{+}$and $\mathrm{C}_{5} \mathrm{~B}_{5}{ }^{-}$chains. The red color corresponds to A segments, the blue color to both $\mathrm{B}^{+}$and $\mathrm{B}^{-}$segments and the green color to $\mathrm{C}$ segments. (b) Radial density profiles of bead $i$ from the gravity center, $\rho_{i}^{\text {rel }}(r)$ for the associate with the weight-average association number, $\left.<A_{\mathrm{S}, \mathrm{W}}\right\rangle=11 ; \rho_{i}^{\mathrm{rel}}(r)=\rho_{i}(r) / \rho$ and $\rho=3$. The red curve (left axis) describes the ensemble- and angular-average density profile of hydrophobic beads $A$, the full and dashed blue curves (right axis) show the densities of the positively and negatively charged PE blocks, respectively and the green curve (right axis) represents the density profile of the $\mathrm{C}$ beads. The full and dashed black curves (right axis) correspond to the positively and negatively charged counterions, respectively. The black dotted curve (left axis) corresponds to the solvent. The insert in Figure $7 \mathrm{~b}$ depicts the difference between the density of the positive and negative charges on the PE chains (dashed curve) and the total charge density profile including small ions (solid curve).

Figure $7 \mathrm{~b}$ depicts the radial density profiles of components from the gravity center for the associate corresponding to the weight-average association number, $\left\langle A_{\mathrm{S}}\right\rangle_{\mathrm{w}}=11$. In this paper and in the Supporting Information, the local density of the DPD beads is generally 
depicted as density relative to the system density $\rho_{i}^{\text {rel }}(r)=\rho_{i}(r) / \rho$. The angularly averaged radial density profiles provide comprehensive picture of the structure of spherical particles, but it could impoverish information on the shape of non-spherical particles. Therefore, we evaluated also the components of the gyration tensor of all micellar systems studied. They are presented in the Supporting Information (see the description of individual figures in Results and Discussion together with corresponding figures in the Supporting Information). The comparison of principal components of the gyration tensor proves that the studied onion micelles are on average spherical particles, even though instantaneous fluctuations in their size exist depending on the solubility, flexibility and consequent mobility of individual chains.

The red curve (left axis), which describes the ensemble-average and angular-average density profile of hydrophobic beads $\mathrm{A}$ in associates with $\left\langle A_{\mathrm{S}}\right\rangle_{\mathrm{w}}=11$, demonstrates the formation of dense insoluble cores in the central part of associates. The cores are small because the associates contain a relatively small number of copolymer chains. The full and dashed blue curves (right axis) show the densities of the positively and negatively charged PE blocks, respectively. Both PE densities are low (the scale on the right axis is amplified almost ten times with respect to the right axis) because the IPEC complex is highly solvated - see the dotted black curve, which depicts the water profile. The dashed curve ( $\mathrm{B}^{-}$beads) is lower than the full one ( $\mathrm{B}^{+}$beads) showing that the composition of the associates, formed in a $1: 1$ mixture of components, is not strictly stoichiometric and the charges on the PE chains do not fully compensate each other. The excess positive charge in the solvated IPEC layer is partially compensated by the counterions (see the blue dashed curve, right axis). This observation is consistent with the experimental measurement of the $\zeta$-potential (see Figure 5). The slight overlap of A and B profiles is mainly the artifact of the ensemble and angular averaging because the shapes of a number of instantaneous associates deviate from ideal spheres ${ }^{41}$. If the associate is, e.g., a prolong ellipsoid, one finds in the intermediate range of $r$ (at the interface between $\mathrm{A}$ and $\mathrm{B}$ domains) either $\mathrm{A}$ or $\mathrm{B}$ beads depending on the direction which one follows. The averaging thus artificially augments the apparent overlap of A and B profiles. The green curve (right axis) representing the density profile of $\mathrm{C}$ beads is low, very broad and strongly overlaps with the blue PE curves because the mutually compatible B and C blocks intermix. The Insert in Figure $7 \mathrm{~b}$ depicts the difference between the densities of the positive and negative charges on PE chains (dashed curve) and the full curve shows the total charge density profile (including small ions). The differences between the densities of the opposite 
charges on the PE chains in the middle layer are larger than the overall deviations from electroneutrality because some small counterions penetrate into this layer, but the imbalance of electric charges is very small. The integration of the positive peak on the full curve in the $r$ region from 2 to 4 yields the total charge smaller than $+0.2 e$. The oscillations are simulation artifacts and do not have any physical meaning. The charge density, $\rho_{\mathrm{q}}$, is evaluated from differences between the numbers of positive and negative charges in narrow spherical layers of the thickness $\Delta r$. The numbers are fairly low and vary by \pm 1 . The maximum charge density is only 0.2 and the high statistics ensemble average curve is subject to high fluctuations.

In summary, the study of the system with soluble PE backbones shows that complex electrostatically stabilized associates are formed in stoichiometric mixtures of components in aqueous media. The associates have a small association number and coexist in equilibrium with non-negligible fractions of free unimer. Their irregular and fast fluctuating IPEC domains surrounding insoluble A cores are highly hydrated and intermix with the $\mathrm{C}$ blocks. The outermost micellar periphery contains predominantly $\mathrm{C}$ beads, but, as is documented by a typical snapshot of the simulation box (Insert in Figure 7a), the overall shape of the associates and their structure do not correspond to onion micelles with segregated domains. The density of the soluble shells is very low and the associates are reminiscent of irregular nano-gel particles.

The main goal of the first series of simulation is investigation of the PE backbone hydrophobicity effect. The results of simulations for $\Delta a_{\mathrm{BS}}=\Delta a_{\mathrm{BC}}=\Delta a_{\mathrm{BI}}=0,2.5,3.5$ and 5 , are compared in the Supporting Information. (Figures S3 to S6). Here we reproduce only the results for the most hydrophobic system with $\Delta a_{\mathrm{BS}}=5$ in Figure 8 . The structure of the Figures and all the parameters of the studied systems, except $\Delta a_{\mathrm{BS}}=\Delta a_{\mathrm{BC}}=\Delta a_{\mathrm{B}-\mathrm{CI}}$, are the same as in Figure 7.

Comparison of the curves in Figures $7 \mathrm{a}$ and $8 \mathrm{a}$ and a more detailed comparison in Figures S3 to S6 reveal the following trends. The association numbers of the parent micelles (see the dashed curves with circles in Figures 7a and 8a and in Figures S3a to S6a) change only slightly with an increase of the hydrophobicity of the PE backbone (in the range of studied $\Delta a_{\mathrm{BS}}$ values). The negligible effect of $\Delta a_{\mathrm{BS}}$ is understandable, because the highly ionized shell-forming PE chains are able to stabilize the core-shell micelles well even if the backbone is moderately hydrophobic. As the hydrophobicity of block A and the length of both blocks are constant in all the systems and the theory predicts that the association number is 
controlled mainly by the properties of block $\mathrm{A}^{107}$, the association number of parent micelles remains almost constant. While the hydrophobicity of the PE backbone does not almost affect the amphiphilic self-assembly of the parent $\mathrm{A}_{5} \mathrm{~B}_{5}{ }^{+}$copolymers, it strongly influences the electrostatic co-assembly of core-shell $\mathrm{A}_{5} \mathrm{~B}_{5}{ }^{+}$micelles with $\mathrm{C}_{5} \mathrm{~B}_{5}{ }^{-}$chains. The increase in the PE hydrophobicity strongly promotes the co-assembly. The distribution functions of the association numbers of the onion micelles shift towards higher $A_{\mathrm{s}}$ and become narrower (see the solid curve with filled circles in Figure 8a and Figures S3a to S6a). In contrast to the system with $\Delta a_{\mathrm{BS}}=0$, typical snapshots for $\Delta a_{\mathrm{BS}}=2.5,3.5$ and 5 reveal the formation of distinct compact nanoparticles with a three-layer structure, which develops and improves along with the increase in $\Delta a_{\mathrm{BS}}$.

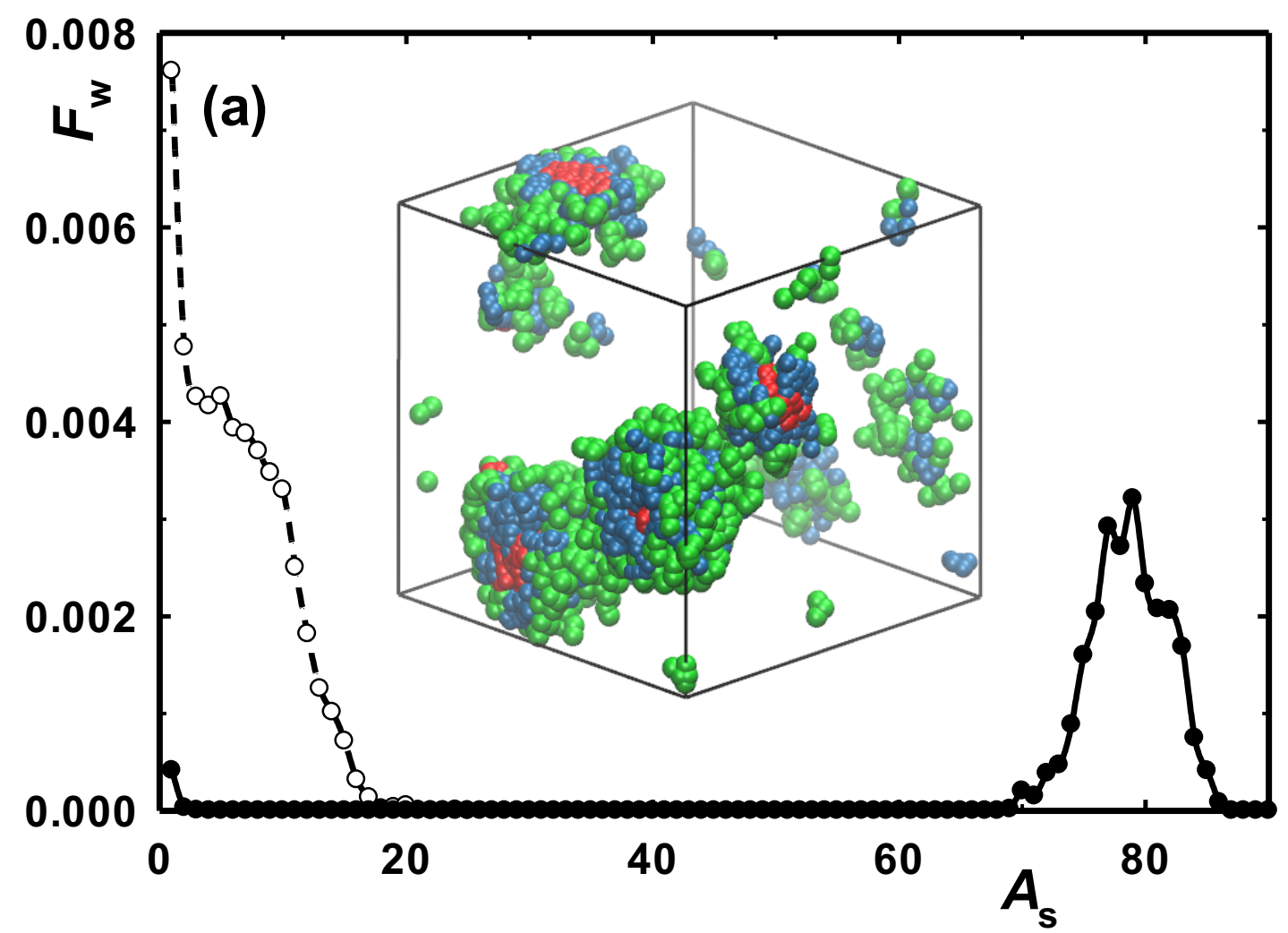




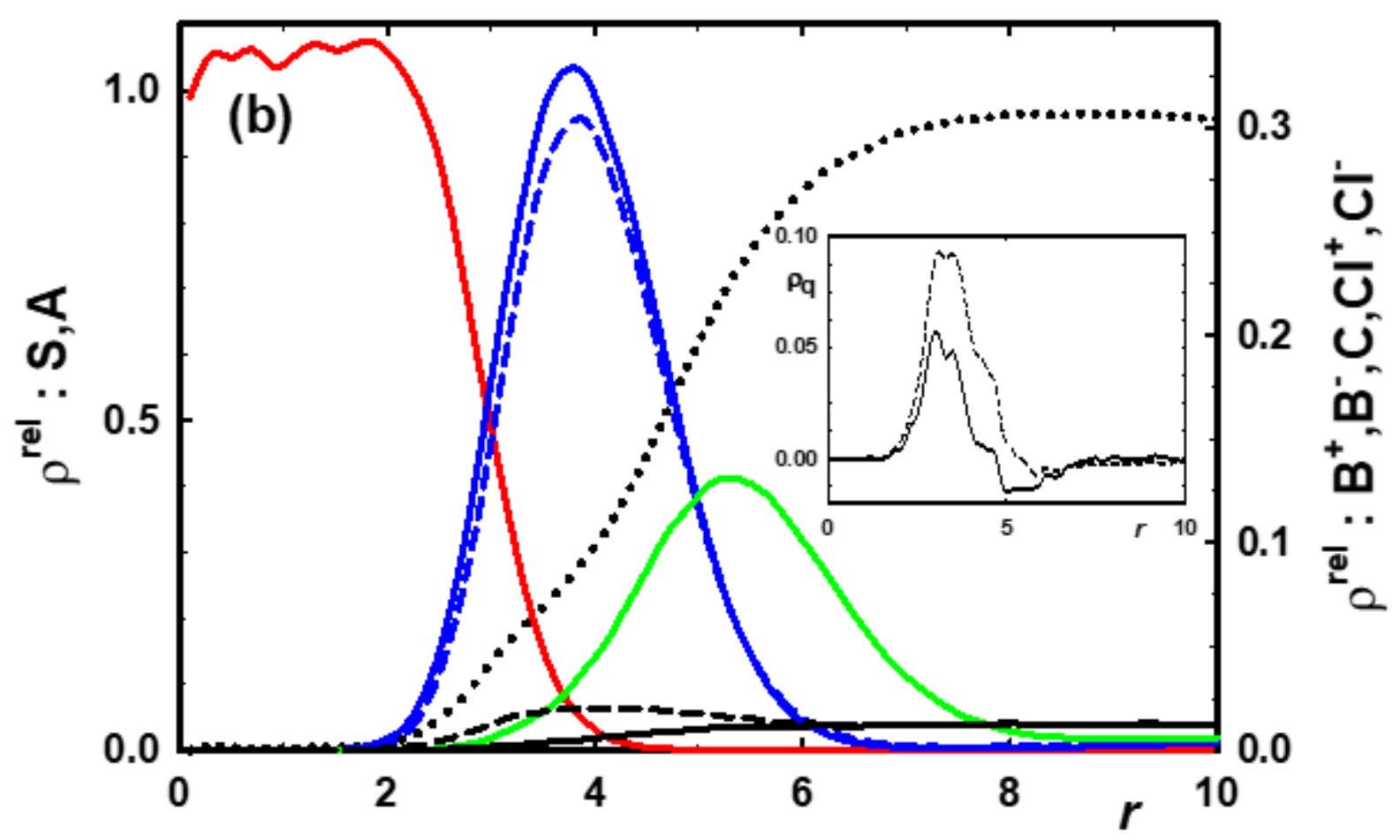

Figure 8. (a) Weight distributions functions, $F_{\mathrm{w}}\left(A_{\mathrm{s}}\right)$, of association numbers $A_{\mathrm{s}}$ in System I with low hydrophilicity of the PE backbone and low compatibility with blocks $\mathrm{C}$ and with counterions for $\Delta a_{\mathrm{BS}}=\Delta a_{\mathrm{BC}}=\Delta a_{\mathrm{BI}}=5$. The inserts show typical snapshot of associates formed in the simulation box. (b) Radial density profiles of components from the gravity center for the associate with the weight-average association number $\left.<A_{\mathrm{S}, \mathrm{W}}\right\rangle=79$ in systems with $\Delta a_{\mathrm{BS}}=\Delta a_{\mathrm{BC}}=\Delta a_{\mathrm{BI}}=5$. The Insert in Figure $8 \mathrm{~b}$ depicts the difference between the density of the positive and negative charges on PE chains and the total charge density profile including small ions. The colors and notation are the same as in Figure 7.

Figure $8 \mathrm{~b}$ and Figures $\mathrm{S} 3 \mathrm{~b}$ to $\mathrm{S} 6 \mathrm{~b}$ show the ensemble-average radial density profiles of individual components (including water and ions) as functions of the distance $r$ from the gravity center of the associate. The comparison of red curves corresponding to hydrophobic blocks $\mathrm{A}$ in Figures $7 \mathrm{~b}$ and $8 \mathrm{~b}$ (and in Figures $\mathrm{S} 3 \mathrm{~b}$ to $\mathrm{S} 6 \mathrm{~b}$ in the Supporting Information) reveals that the size of the hydrophobic cores grows with increasing $\Delta a_{\mathrm{BS}}$. This means that the numbers of $\mathrm{A}_{5} \mathrm{~B}_{5}{ }^{+}$chains which form compact inner cores of composed associates increase in contrast to parent micelles, whose association number almost does not depend on $\Delta a_{\mathrm{BS}}$. The changes in the blue curves (for charged B blocks) and in their overlap with the green curves for the $\mathrm{C}$ blocks show that the density of the PE beads in the middle layer and the segregation of $\mathrm{B}$ and $\mathrm{C}$ increase and the IPEC hydration decreases with $\Delta a_{\mathrm{BS}}$. Simultaneously, the difference between the numbers of $\mathrm{B}^{+}$and $\mathrm{B}^{-}$beads in the middle layer and the concentration of counterions in the IPEC layer diminishes. The Insert shows low charge density of the IPEC layer. 
As already mentioned, in Figure S7 of the Supporting Information, we present the principal components of the gyration tensor, $R_{g, \alpha}^{2}(\alpha=1,2,3)$, of associates for System I with low and high $\mathrm{B}$ hydrophobicity. We see that $R_{g, 1}^{2} \cong R_{g, 2}^{2} \cong R_{g, 3}^{2}$ which indicates that the associates are effectively spherical. To show how the expansion of PE chains differ in parent micelles and in the compact middle IPEC layer, in Figure S8 of the Supporting Information, we compare the square end-to-end distances of $\mathrm{B}^{+}$blocks, $R_{e, \mathrm{~B}}^{2}$, in associates and in parent $\mathrm{A}_{5} \mathrm{~B}_{5}$ systems. In parent micelles, the ionized $\mathrm{B}^{+}$blocks are fairly stretched and the expansion is promoted by the hydrophilicity of PE backbone. The $R_{e, \mathrm{~B}}^{2}$ values generally increase with $A_{\mathrm{S}}$ due to increasing crowding and electrostatic repulsion of the PE chains in micellar shells. Some "oscillation-like" fluctuations (mainly for high $\mathrm{As}_{\mathrm{S}}$ ) are due to low fractions of high aggregates and consequent low statistics. As expected, the expansion of $\mathrm{B}^{+}$blocks in free unimer chains $\left(A_{\mathrm{S}}=1\right)$ depends on the hydrophobicity of PE backbone. The comparison shows that the chains in dense IPEC layers are generally less expanded than those in soluble shells of parent micelles and that $R_{e, \mathrm{~B}}^{2}$ values decrease with the increase in $a_{B S}$, which means that the middle layer formed by a more hydrophobic PE is narrower than that formed by a hydrophilic PE. However, critical comparison of relevant structural parameters reveals that the high density of IPEC layers in systems with fairly hydrophobic PE backbones is mainly the result of important interpenetration of $\mathrm{B}^{+}$and $\mathrm{B}^{-}$blocks and their low hydration.

In summary, the most important feature of the behavior of stoichiometric systems with the same lengths of all the blocks is the promotion of co-assembly with worsening solubility of the PE blocks. Both the association numbers and fraction of large associates appreciably increase. In systems with slightly increased hydrophobicity of PE blocks, the associates adopt the onion structure. If the lengths of blocks are equal or comparable and B blocks are at least slightly hydrophobic, the layered associates contain higher numbers of $\mathrm{A}_{5} \mathrm{~B}_{5}{ }^{+}$(and consequently also of $\mathrm{C}_{5} \mathrm{~B}_{5}{ }^{-}$) chains compared with parent core-shell $\mathrm{A}_{5} \mathrm{~B}_{5}{ }^{+}$micelles formed under the same conditions. The increase in total $A_{\mathrm{S}}$ of onion micelles and in the number of $\mathrm{A}_{5} \mathrm{~B}_{5}{ }^{+}$can be easy explained: The stabilization of micelles containing not only the insoluble A core but also an insoluble IPEC layer requires increased numbers of soluble shell-forming chains. The simulation shows that the ratio of components in stoichiometric onion micelles corresponds roughly to that in the solution (ca. 1:1), which means that the ratio of soluble to insoluble beads, $r_{\mathrm{S}-\mathrm{I}}$ decreases from 1.0 in parent micelles to 0.33 in onion micelles and in this case, the theory of amphiphilic block copolymer association predicts larger $A_{\mathrm{S}}$ than that of the parent micelles. ${ }^{22}$ As the numbers of $\mathrm{B}^{+}$and $\mathrm{B}^{-}$(i.e., numbers of $\mathrm{AB}^{+}$and $\mathrm{B}^{-} \mathrm{C}$ components) 
are equal, the increase in $A_{\mathrm{S}}$ requires an increase in the number of $\mathrm{AB}^{+}$chains. This observation is very important from the experimental point of view because it shows that in reversible (kinetically non-frozen) systems, a non-negligible reorganization and fairly massive redistribution of chains of both types among associates occur upon mixing the solution of parent micelles with the oppositely charged copolymer.

\section{Systems II and III}

In the last part, we addressed the effect of the length of the blocks. As the simulations are very time consuming, we selected two systems only: System II and System III (see Table 2) which, as we believe, demonstrate important trends in the behavior. In System II consisting of 240 $\mathrm{A}_{5} \mathrm{~B}_{5}{ }^{+}$and 240 of $\mathrm{C}_{7} \mathrm{~B}_{5}{ }^{-}$chains, we expect better stabilization of associates because the neutral water-soluble block $\mathrm{C}$ is longer, while the lengths of the insoluble block $\mathrm{A}$ and of both $\mathrm{PE}$ blocks $\mathrm{B}^{+}$and $\mathrm{B}^{-}$are the same as in the previous system, i.e., these blocks are shorter than the $\mathrm{C}$ block. Hence we assume that the association numbers will be lower compared to the previous system, but the formation of the IPEC layer (including the ratio of oppositely charged components and the concentration of counterions) will be similar. The weight distribution functions of association numbers of onion micelles are presented in Figure 9. They confirm our expectations concerning $A_{\mathrm{S}}$. Compared with the previously discussed systems, the peaks of the associates are broad and their positions shift to lower $A_{\mathrm{S}}$. The micellar peak in the system with $\Delta a_{\mathrm{BS}}=0$ is only insignificantly separated from the peak of unimers and low associates (dimers and trimers). It is broader than that in the corresponding parent system and extends to higher $A_{\mathrm{s}}$, but the maxima of the peaks of onion and parent micelles are close to each other on the $A_{\mathrm{S}}$ scale, which indicates that the number of $\mathrm{A}_{5} \mathrm{~B}_{5}{ }^{+}$ chains in the onion micelles is lower than their number in the parent micelles. Better solubility, i.e., higher mobility of copolymer chains facilitates the simulations and the curves depicting the weight fractions of associates as a function of $A_{\mathrm{S}}$ are smoother and more symmetrical as compared with System I.

Radial density profiles for two systems with $\Delta a_{\mathrm{BS}}=3.5$ and 5 are shown in the Supporting Information (Figures $\mathrm{S} 9$ and $\mathrm{S} 10$ ). As the $\mathrm{C}_{7}$ blocks are longer than the $\mathrm{B}_{5}$ blocks, they are more expanded and reach to longer distances from the gravity centers of the associates than the $\mathrm{C}_{5}$ blocks in the previous systems, which promotes their segregation from the IPEC layer even for the relatively hydrophilic PE block $\left(\Delta a_{\mathrm{BS}}=3.5\right)$ when the IPEC layer is strongly hydrated and can relatively easily intermix with C. Soluble chains $\mathrm{C}$ try to maximize the number of their conformations (entropy) and exploit the dilute periphery of the 
associates. This is demonstrated by the fairly segregated density profiles of associates with nonzero, albeit still low, $\Delta a_{\mathrm{BS}}$ and also by snapshots of the simulation box. A typical snapshot (1.h.s. Insert in Figure 9) depicts well-developed onion micelles formed in the system with only few hydrophobic PE blocks with $\Delta a_{\mathrm{BS}}=3.5$. The r.h.s. insert shows a snapshot for $\Delta a_{\mathrm{BS}}=5$. In this case, the three-layer structure is better developed, but both snapshots confirm the formation of onion micelles.

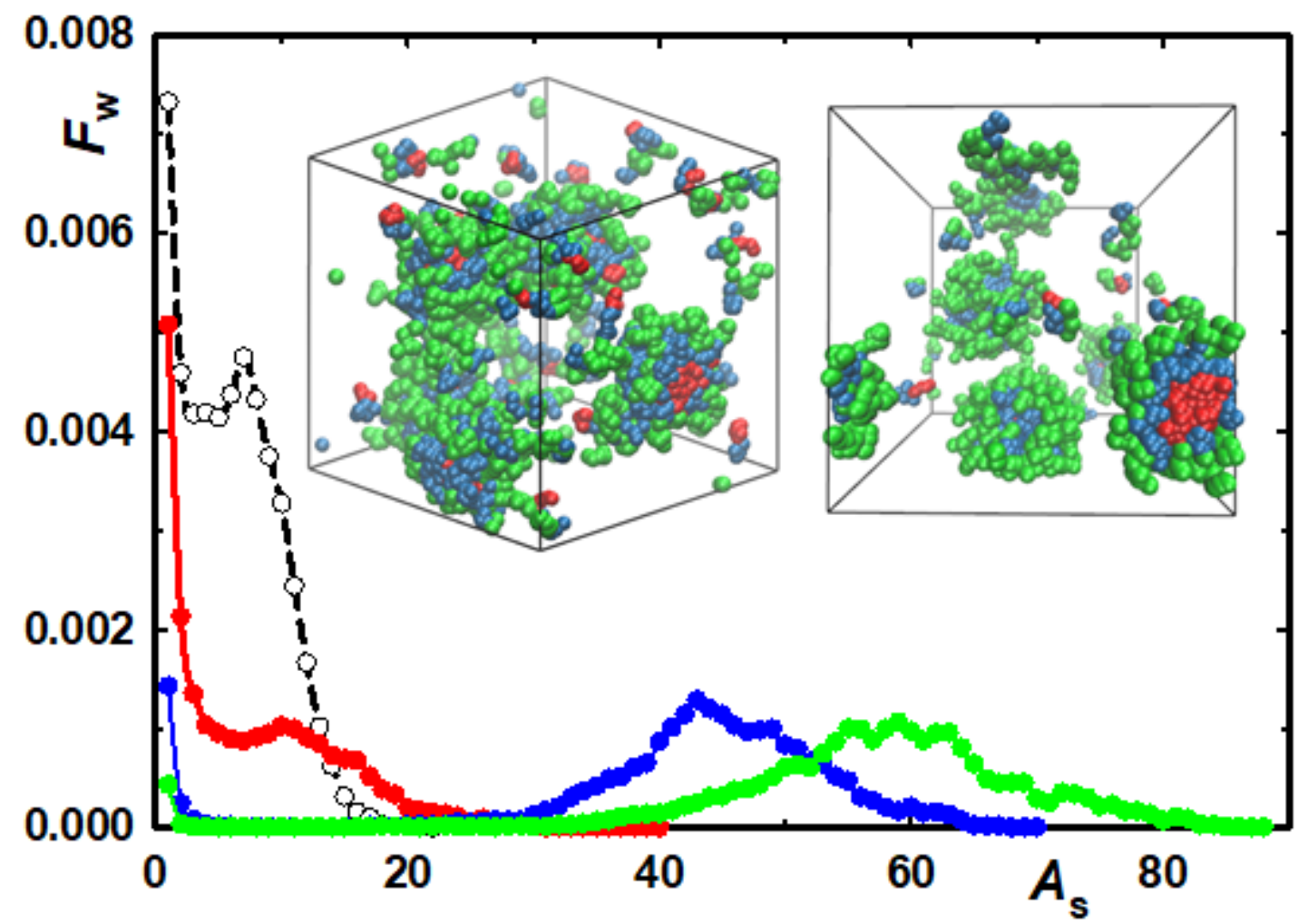

Figure 9. Weight distribution functions, $F_{\mathrm{w}}\left(A_{\mathrm{s}}\right)$, of association numbers $A_{\mathrm{s}}$ for parent micelles $\mathrm{A}_{5} \mathrm{~B}_{5}{ }^{+}$with $\Delta a_{\mathrm{BS}}=0$ (black dashed curve) and in System II for $\Delta a_{\mathrm{BS}}=\Delta a_{\mathrm{BC}}=\Delta a_{\mathrm{BI}}=0$ (red curve), 3.5 (blue curve) and 5 (green curve). The 1.h.s. insert shows a typical snapshot of associates for associates with $\Delta a_{\mathrm{BS}}=\Delta a_{\mathrm{BC}}=\Delta a_{\mathrm{BI}}=3.5$ and r.h.s. snapshot for a value of 5 .

The last studied System III is composed of $240 \mathrm{~A}_{5} \mathrm{~B}_{5}{ }^{+}$and $400 \mathrm{C}_{5} \mathrm{~B}_{3}{ }^{-}$chains, i.e., the numbers of chains differ, but the numbers of positively and negatively charged beads are the same $(Z=1)$. Based on a simple electrostatic consideration and on the results of the previous simulations, it is not surprising that roughly neutral (in fact, slightly charged) associates formed in mixtures with $\mathrm{Z}=1$ contain an excess of $\mathrm{C}_{5} \mathrm{~B}_{3}{ }^{-}$chains and their shells are formed by fairly large numbers of water-soluble $\mathrm{C}_{5}$ blocks. Such onion micelles are, of course, readily soluble. The distribution functions of the association numbers shown in Figure 10 are lower than those in System I. As the radial density profiles for non-zero $\Delta a_{\mathrm{BS}}$ are qualitatively very similar to each, we present them only in the Supporting Information (Figure S9 for System II 
and Figure S10 for System III). The shapes of the curves indicate a three-layer onion structure with three segregated domains. Insoluble cores A are smaller than those of micelles in corresponding system I, but the $\mathrm{C}$ shells are relatively crowded and therefore quite expanded. Blocks C extend to distances comparable with System I (with large cores) and in only slightly shorter distances than in System II (with longer $C_{7}$ chains). The inserts in Figure 10 show typical associates for $\Delta a_{\mathrm{BS}}=3.5$ and 5. As $A_{\mathrm{S}}$ values for given $\Delta a_{\mathrm{BS}}$ are lower than those in System I and System II, the number of onion micelles is higher and the snapshots are fairly crowded and slightly less comprehensive.

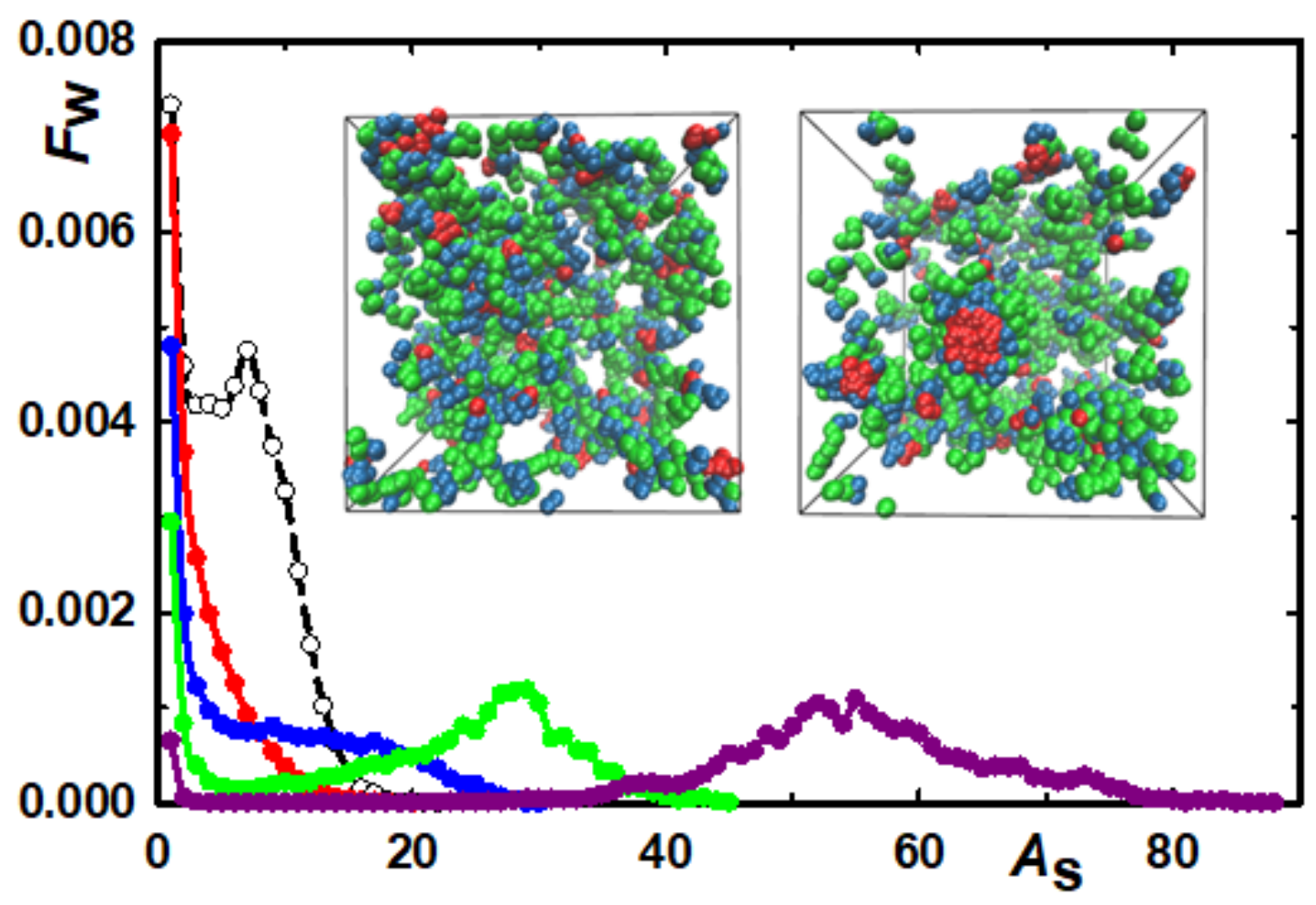

Figure 10. Weight distributions functions, $F_{\mathrm{w}}\left(A_{\mathrm{s}}\right)$, of association numbers $A_{\mathrm{s}}$ for parent micelles $\mathrm{A}_{5} \mathrm{~B}_{5}{ }^{+}$with $\Delta a_{\mathrm{BS}}=0$ (black dashed curve) and in System III for $\Delta a_{\mathrm{BS}}=\Delta a_{\mathrm{BC}}=\Delta a_{\mathrm{BI}}=0$ (red curve), 3.5 (blue curve), 5 (green curve) and 7.5 (cyan curve). The 1.h.s. insert shows a typical snapshot of associates with $\Delta a_{\mathrm{BS}}=\Delta a_{\mathrm{BC}}=\Delta a_{\mathrm{BI}}=3.5$ and r.h.s. snapshot for a value of 5 .

Finally we analyzed the shape of onion micelles formed by components with different lengths of blocks. In Figures S11 and S12 of the Supporting Information, we show the principal components of the gyration tensor, $R_{g, \alpha}^{2}(\alpha=1,2,3)$ as a function of $A_{\mathrm{s}}$ for System II and System III with low and high values of the hydrophobicity. The inspection of curves shows that $R_{g, 1}^{2} \cong R_{g, 2}^{2} \cong R_{g, 3}^{2}$ and confirms that these associates have spherical shape 


\section{Summary and Conclusions}

We used a combination of several experimental methods (scattering and imaging techniques) to study the formation of three-layer (onion) polymeric micelles in aqueous media by sequential application of amphiphilic self-assembly of a copolymer containing one hydrophobic block and one polyelectrolyte block and electrostatic co-assembly of core-shell micelles with polyelectrolyte shell (prepared in the first step) with a double-hydrophilic copolymer composed of a neutral water-soluble block and an oppositely charged PE block. The study was motivated by the following controversy: In spite of the fact that thermodynamic analysis predicts the formation of onion micelles as a favorable process, there exist very few publications reporting successful attempts at their preparation. ${ }^{44,} 47,59$ Because we are of the opinion that the experimental difficulties are of a kinetic origin, we analyzed potential obstacles and selected a system in which we did not expect insurmountable kinetic barriers (relatively short and flexible chains, low $T_{\mathrm{G}}$, moderate hydrophobicity of PE backbones, etc.). Experimental study confirmed that stable aqueous dispersion onion micelles can be prepared by a combination of polymer self- and co-assembly.

The extensive simulation study (DPD with explicit electrostatics) was aimed at decisive trends of the self- and co-assembling behavior under the assumption that the kinetic barriers are low and do not hinder the equilibration. As DPD simulations with explicit electrostatics are time-consuming, we focused on stoichiometric systems with matched positive and negative charges of PE chains and investigated mainly the effects of (i) the length of different blocks and (ii) the hydrophobicity of the polyelectrolyte backbones on the association number, fraction of associates and their structure.

To prove that in the systems under consideration, the equilibrium (minimum energy) polymeric species are fully equilibrated associates composed of $A_{5} B_{5}{ }^{+}$and $B_{n}{ }^{-} C_{m}$ chains, we did not simulate the sequential assembly, but started the simulations from different initial conditions: mostly from a random mixture of non-associated chains of both types, but also from equilibrated conformations formed in systems with different hydrophobicity of B blocks. The simulations from different initial conditions converged to the same final state and confirmed our working hypothesis. The modeling performed for a set of interaction parameters emulating polymers with different hydrophobicity of the PE backbones, unambiguously showed that the fully equilibrated states are dispersions of layered spherical associates containing both copolymer components. 
The simulation results show that the formation of onion micelles with reasonably segregated layers requires, similarly to the electrostatic co-assembly of simpler $\mathrm{C}_{m} \mathrm{~B}_{n}{ }^{+}$and $\mathrm{C}_{m} \mathrm{~B}_{n}{ }^{-}$core/shell micelles which we studied earlier ${ }^{41}{ }^{43}$, some hydrophobicity of PE backbones and some incompatibility of the B and C blocks. The hydrophobicity of B and incompatibility of $\mathrm{B}$ and $\mathrm{C}$ do not have to be large, values of $\Delta a_{\mathrm{BS}}=\Delta a_{\mathrm{BS}}=2.5$ are sufficient but the IPEC layer is in this case strongly solvated and has a fairly low density. The increase in $\Delta a_{\mathrm{BS}}$ and $\Delta a_{\mathrm{BC}}$ promotes the formation of well-defined onion structures with dense and reasonably segregated layers. The associates formed in systems with $\Delta a_{\mathrm{BS}}=\Delta a_{\mathrm{BS}}=0$ are nanogel-like particles containing dense A domains and irregular low density IPEC domains which are strongly hydrated and intermixed with $\mathrm{C}$ blocks.

The comparison of association numbers of $\mathrm{A}_{n} \mathrm{~B}_{n}{ }^{+}$and $\mathrm{A}_{n} \mathrm{~B}_{n}{ }^{+} / \mathrm{C}_{m} \mathrm{~B}_{n}{ }^{-}$(onion) micelles ${ }^{96}$ formed under the same conditions shows that the numbers of $\mathrm{AB}$ chains in both types of micelles differ, which is important from the experimental point of view: it indicates that, during the sequential preparation of onion micelles in kinetically non-frozen systems, a considerable redistribution of the $\mathrm{AB}$ chains among the micelles takes place. This could be the reason why we did not succeed in preparing stable dispersions of onion micelles based on parent micelles with kinetically frozen polystyrene cores (unpublished data).

Simulations also confirm the theoretical prediction that the total association number (sum of both types of chains) is controlled by the ratio of insoluble-to-soluble beads, $r_{\text {S-I }}$. Parent micelles contain insoluble beads A only, but the number of insoluble beads in onion micelles is the sum of $\mathrm{A}_{1} \mathrm{~B}^{+}$and $\mathrm{B}^{-}$beads. Depending on relative lengths of $\mathrm{A}, \mathrm{B}^{+}, \mathrm{B}^{-}$and $\mathrm{C}$ blocks, the ratio $r_{\mathrm{S}-\mathrm{I}}$ in electrically neutral 1:1 onion micelles can be either higher or lower than that in parent micelles. The increase in $r_{\mathrm{S}-\mathrm{I}}$ (under the condition of particle electroneutrality) provokes the decrease in the number of $\mathrm{AB}^{+}$chains in central cores of onion micelles and vice versa. Total association number generally increases during onion micelle formation, but much less in the former case than in the latter case. Even though the simulations were performed in a limited range of relative lengths of blocks, they help to understand the slightly surprising observation that onion micelles in the experimentally studied system are smaller than the parent micelles.

In the experimental part, we studied a special case, in which the lengths of both blocks $\mathrm{B}^{-}$and $\mathrm{C}$ in the second copolymer are appreciably longer than both blocks $\mathrm{A}$ and $\mathrm{B}^{+}$in the first (parent micelles-forming) copolymer. In this case, the onions with 1:1 matched electric charges contain 5.8 times lower number of chains of the second copolymer, however $r_{\mathrm{S}-\mathrm{I}}$ 
increases from 0.7 (parent micelles) to 1.5 (onion micelles). As a consequence, micelles with a small central core formed by a low number of A blocks (strongly hydrophobic PLA) covered by a thin and compact IPEC layer of moderately hydrophobic PEs and stabilized by a shell of soluble C blocks formed in aqueous media. As the "tethering" density of $\mathrm{C}$ blocks (water-soluble PEO) to the IPEC surface is low, inner parts of PEO chains recoil back towards the insoluble IPEC shell to create a relatively dense inner shell protecting the IPEC/shell interface from interacting with solvent (water). The extrapolation of co-assembling trends observed in simulations indicates that in this special case, the size onion micelles can be smaller than that of parent micelles with expanded PE shells. Even though the changes in numbers of chains forming the inner core are a general feature of studied systems, the decrease in size of onion micelles with respect to parent micelles is a specific property of the experimentally studied system only.

\section{Acknowledgement}

This study was supported by the Czech Science Foundation (Grant No. 20-01233S). MS and ML acknowledge funding from the European Union's Horizon 2020 research and innovation programme (Project VIMMP: Virtual Materials Marketplace, No. 760907), from the ERDF/ESF project UniQSurf-Centre of biointerfaces and hybrid functional materials (no. CZ.02.1.01/0.0/0.0/17_048/0007411), and from the Internal Grant Agency of Jan Evangelista Purkyně University in Ústí nad Labem (project no. UJEP-SGS-2019-53-005-3). MŠ acknowledges support from the Ministry of Education, Youth and Sports of the Czech Republic (Operational Programme Research, Development and Education: "Excellent Research Teams", Project No. CZ.02.1.01/0.0/0.0/15_003/0000417-CUCAM.

\section{References}

1. Avsar, S. Y.; Kyropoulou, M.; Di Leone, S.; Schoenenberger, C. A.; Meier, W. P.; Palivan, C. G. Biomolecules Turn Self-Assembling Amphiphilic Block Co-polymer Platforms Into Biomimetic Interfaces. Frontiers in Chemistry 2019, 6, DOI:

10.3389/fchem.2018.00645.

2. Kataoka, K.; Harada, A.; Nagasaki, Y. Block copolymer micelles for drug delivery: Design, characterization and biological significance. Advanced Drug Delivery Reviews 2012, 64, 37-48 DOI: 10.1016/j.addr.2012.09.013.

3. Blanazs, A.; Armes, S. P.; Ryan, A. J. Self-Assembled Block Copolymer Aggregates: From Micelles to Vesicles and their Biological Applications. Macromolecular Rapid Communications 2009, 30 (4-5), 267-277 DOI: 10.1002/marc.200800713. 
4. Kabanov, A. V.; Alakhov, V. Y. Pluronic (R) block copolymers in drug delivery: From micellar nanocontainers to biological response modifiers. Critical Reviews in Therapeutic Drug Carrier Systems 2002, 19 (1), 1-72 DOI:

10.1615/CritRevTherDrugCarrierSyst.v19.i1.10.

5. Gohy, J.-F.; Zhao, Y. Photo-responsive block copolymer micelles: design and behavior. Chemical Society Reviews 2013, 42 (17), 7117-7129 DOI: 10.1039/c3cs35469e.

6. Walther, A.; Mueller, A. H. E. Janus Particles: Synthesis, Self-Assembly, Physical Properties, and Applications. Chemical Reviews 2013, 113 (7), 5194-5261 DOI: $10.1021 / \mathrm{cr} 300089 \mathrm{t}$.

7. Mai, Y. Y.; Eisenberg, A. Self-assembly of block copolymers. Chemical Society Reviews 2012, 41 (18), 5969-5985 DOI: 10.1039/c2cs35115c.

8. Moughton, A. O.; Hillmyer, M. A.; Lodge, T. P. Multicompartment Block Polymer Micelles. Macromolecules 2012, 45 (1), 2-19 DOI: 10.1021/ma201865s.

9. O'Reilly, R. K.; Hawker, C. J.; Wooley, K. L. Cross-linked block copolymer micelles: functional nanostructures of great potential and versatility. Chemical Society Reviews 2006, 35 (11), 1068-1083 DOI: 10.1039/b514858h.

10. Forster, S.; Plantenberg, T. From self-organizing polymers to nanohybrid and biomaterials. Angewandte Chemie-International Edition 2002, 41 (5), 689-714.

11. Forster, S.; Hermsdorf, N.; Bottcher, C.; Lindner, P. Structure of polyelectrolyte block copolymer micelles. Macromolecules 2002, 35 (10), 4096-4105 DOI: 10.1021/ma011565y.

12. Rud, O. V.; Mercurieva, A. A.; Leermakers, F. A. M.; Birshtein, T. M. Collapse of Polyelectrolyte Star. Theory and Modeling. Macromolecules 2012, 45 (4), 2145-2160 DOI: $10.1021 / \mathrm{ma} 202201 \mathrm{~m}$.

13. Zhulina, E. B.; Borisov, O. V. Theory of Block Polymer Micelles: Recent Advances and Current Challenges. Macromolecules 2012, 45 (11), 4429-4440 DOI:

10.1021/ma300195n.

14. Voets, I. K.; Leermakers, F. A. M. Self-consistent field theory for obligatory coassembly. Physical Review E 2008, 78 (6), DOI: 10.1103/PhysRevE.78.061801.

15. Stepanek, M.; Krijtova, K.; Prochazka, K.; Teng, Y.; Webber, S. E.; Munk, P. Solubilization and release of hydrophobic compounds from block copolymer micelles. I. Partitioning of pyrene between polyelectrolyte micelles and the aqueous phase. Acta Polymerica 1998, 49 (2-3), 96-102.

16. Zhang, L. F.; Eisenberg, A. Formation of crew-cut aggregates of various morphologies from amphiphilic block copolymers in solution. Polymers for Advanced Technologies 1998, 9 (10-11), 677-699.

17. Walther, A.; Muller, A. H. E. Janus particles. Soft Matter 2008, 4 (4), 663-668 DOI: 10.1039/b718131k. 
18. Gohy, J. F.; Lohmeijer, B. G. G.; Alexeev, A.; Wang, X. S.; Manners, I.; Winnik, M. A.; Schubert, U. S. Cylindrical micelles from the aqueous self-assembly of an amphiphilic poly(ethylene oxide)-b-poly(ferrocenylsilane) (PEO-b-PFS) block copolymer with a metallosupramolecular linker at the block junction. Chemistry-a European Journal 2004, 10 (17), 4315-4323 DOI: 10.1002/chem.200400222.

19. Liu, F. T.; Eisenberg, A. Preparation and $\mathrm{pH}$ triggered inversion of vesicles from poly(acrylic acid)-block-polystyrene-block-poly(4-vinyl pyridine). Journal of the American Chemical Society 2003, 125 (49), 15059-15064 DOI: 10.1021/ja038142r.

20. Chen, L.; Shen, H. W.; Eisenberg, A. Kinetics and mechanism of the rod-to-vesicle transition of block copolymer aggregates in dilute solution. Journal of Physical Chemistry $B$ 1999, 103 (44), 9488-9497 DOI: 10.1021/jp9913665.

21. Shen, H. W.; Eisenberg, A. Morphological phase diagram for a ternary system of block copolymer PS310-b-PAA(52)/dioxane/H2O. Journal of Physical Chemistry B 1999, 103 (44), 9473-9487 DOI: 10.1021/jp991365c.

22. Nagarajan, R.; Ruckenstein, E. Theory of surfactant self-assembly - a predictive molecular thermodynamic approach. Langmuir 1991, 7 (12), 2934-2969 DOI: 10.1021/la00060a012.

23. Nagarajan, R.; Ganesh, K. Block copolymer self-assembly in selective solvents spherical micelles with segregated cores. Journal of Chemical Physics 1989, 90 (10), 5843 5856 DOI: $10.1063 / 1.456390$.

24. Nagarajan, R.; Ganesh, K. Block copolymer self-assembly in selective solvents theory of solubilization in spherical micelles. Macromolecules 1989, 22 (11), 4312-4325 DOI: 10.1021/ma00201a029.

25. Halperin, A.; Alexander, S. Polymeric micelles - their relaxation kinetics. Macromolecules 1989, 22 (5), 2403-2412 DOI: 10.1021/ma00195a069.

26. Hoven, C. V.; Garcia, A.; Bazan, G. C.; Nguyen, T. Q. Recent Applications of Conjugated Polyelectrolytes in Optoelectronic Devices. Advanced Materials 2008, 20 (20), 3793-3810 DOI: 10.1002/adma.200800533.

27. Prausnitz, M. R.; Langer, R. Transdermal drug delivery. Nature Biotechnology 2008, 26 (11), 1261-1268 DOI: 10.1038/nbt.1504.

28. Thomas, S. W.; Joly, G. D.; Swager, T. M. Chemical sensors based on amplifying fluorescent conjugated polymers. Chemical Reviews 2007, 107 (4), 1339-1386 DOI: $10.1021 / \mathrm{cr} 0501339$.

29. Discher, D. E.; Eisenberg, A. Polymer vesicles. Science 2002, 297 (5583), 967-973 DOI: $10.1126 /$ science. 1074972 .

30. Singh, M.; Briones, M.; Ott, G.; O'Hagan, D. Cationic microparticles: A potent delivery system for DNA vaccines. Proceedings of the National Academy of Sciences of the United States of America 2000, 97 (2), 811-816 DOI: 10.1073/pnas.97.2.811. 
31. Drummond, C. J.; Fong, C. Surfactant self-assembly objects as novel drug delivery vehicles. Current Opinion in Colloid \& Interface Science 1999, 4 (6), 449-456 DOI: 10.1016/s1359-0294(00)00020-0.

32. Lefevre, N.; Fustin, C.-A.; Gohy, J.-F. Polymeric Micelles Induced by Interpolymer Complexation. Macromolecular Rapid Communications 2009, 30 (22), 1871-1888 DOI: 10.1002/marc.200900355.

33. Voets, I. K.; de Keizer, A.; Stuart, M. A. C. Complex coacervate core micelles. Advances in Colloid and Interface Science 2009, 147-48, 300-318 DOI: 10.1016/j.cis.2008.09.012.

34. Stuart, M. A. C.; Hofs, B.; Voets, I. K.; de Keizer, A. Assembly of polyelectrolytecontaining block copolymers in aqueous media. Current Opinion in Colloid \& Interface Science 2005, 10 (1-2), 30-36 DOI: 10.1016/j.cocis.2005.04.004.

35. Berret, J. F.; Vigolo, B.; Eng, R.; Herve, P.; Grillo, I.; Yang, L. Electrostatic selfassembly of oppositely charged copolymers and surfactants: A light, neutron, and X-ray scattering study. Macromolecules 2004, 37 (13), 4922-4930 DOI: 10.1021/ma0498722.

36. van der Burgh, S.; de Keizer, A.; Stuart, M. A. C. Complex coacervation core micelles. Colloidal stability and aggregation mechanism. Langmuir 2004, 20 (4), 1073-1084 DOI: $10.1021 / \mathrm{la} 035012 \mathrm{n}$.

37. Kramarenko, E. Y.; Khokhlov, A. R.; Reineker, P. Micelle formation in a dilute solution of block copolymers with a polyelectrolyte block complexed with oppositely charged linear chains. Journal of Chemical Physics 2003, 119 (9), 4945-4952 DOI: $10.1063 / 1.1594720$.

38. Dobrynin, A. V.; Rubinstein, M. Theory of polyelectrolytes in solutions and at surfaces. Progress in Polymer Science 2005, 30 (11), 1049-1118 DOI:

10.1016/j.progpolymsci.2005.07.006.

39. Rumyantsev, A. M.; Zhulina, E. B.; Borisov, O. V. Scaling Theory of Complex Coacervate Core Micelles. Acs Macro Letters 2018, 7 (7), 811-816 DOI:

10.1021/acsmacrolett.8b00316.

40. Borreguero, J. M.; Pincus, P. A.; Sumpter, B. G.; Goswami, M. Unraveling the Agglomeration Mechanism in Charged Block Copolymer and Surfactant Complexes. Macromolecules 2017, 50 (3), 1193-1205 DOI: 10.1021/acs.macromo1.6b02319.

41. Sindelka, K.; Limpouchova, Z.; Lisal, M.; Prochazka, K. The electrostatic coassembly in non-stoichiometric aqueous mixtures of copolymers composed of one neutral water-soluble and one polyelectrolyte (either positively or negatively charged) block: a dissipative particle dynamics study. Physical Chemistry Chemical Physics 2016, 18 (24), 16137-16151 DOI: 10.1039/c6cp01047d.

42. Goswami, M.; Borreguero, J. M.; Pincus, P. A.; Sumpter, B. G. Surfactant-Mediated Polyelectrolyte Self-Assembly in a Polyelectrolyte-Surfactant Complex. Macromolecules 2015, 48 (24), 9050-9059 DOI: 10.1021/acs.macromol.5b02145. 
43. Sindelka, K.; Limpouchova, Z.; Lisal, M.; Prochazka, K. Dissipative Particle Dynamics Study of Electrostatic Self-Assembly in Aqueous Mixtures of Copolymers Containing One Neutral Water-Soluble Block and One Either Positively or Negatively Charged Polyelectrolyte Block. Macromolecules 2014, 47 (17), 6121-6134 DOI: 10.1021/ma501018x.

44. Lysenko, E. A.; Bilan, R. S.; Chelushkin, P. S. Block-copolymer micelles with a interpolyelectrolyte crown. Polymer Science Series C 2017, 59 (1), 35-48 DOI: 10.1134/s 1811238217010076.

45. Palyulin, V. V.; Potemkin, I. I. Mixed versus ordinary micelles in the dilute solution of AB and BC diblock copolymers. Macromolecules 2008, 41 (12), 4459-4463 DOI: $10.1021 / \mathrm{ma} 8003949$.

46. Humpolickova, J.; Stepanek, M.; Prochazka, K.; Hof, M. Solvent relaxation study of $\mathrm{pH}$-dependent hydration of poly(oxyethylene) shells in polystyrene-block-poly(2vinylpyridine)-block-poly(oxyethylene) micelles in aqueous solutions. Journal of Physical Chemistry A 2005, 109 (48), 10803-10812 DOI: 10.1021/jp053348v.

47. Lysenko, E. A.; Chelushkin, P. S.; Bronich, T. K.; Eisenberg, A.; Kabanov, V. A.; Kabanov, A. V. Formation of multilayer polyelectrolyte complexes by using block ionomer micelles as nucleating particles. Journal of Physical Chemistry B 2004, 108 (33), 1235212359 DOI: $10.1021 / \mathrm{jp} 048777 \mathrm{~s}$.

48. Plestil, J.; Kriz, J.; Tuzar, Z.; Prochazka, K.; Melnichenko, Y. B.; Wignall, G. D.; Talingting, M. R.; Munk, P.; Webber, S. E. Small-angle neutron scattering study of oniontype micelles. Macromolecular Chemistry and Physics 2001, 202 (4), 553-563 DOI: 10.1002/1521-3935(20010201)202:4<553::aid-macp553>3.0.co;2-6.

49. Podhajecka, K.; Stepanek, M.; Prochazka, K.; Brown, W. Hybrid polymeric micelles with hydrophobic cores and mixed polyelectrolyte/nonelectrolyte shells in aqueous media. 2. Studies of the shell behavior. Langmuir 2001, 17 (14), 4245-4250 DOI: 10.1021/la010247p.

50. Stepanek, M.; Podhajecka, K.; Tesarova, E.; Prochazka, K.; Tuzar, Z.; Brown, W. Hybrid polymeric micelles with hydrophobic cores and mixed polyelectrolyte/nonelectrolyte shells in aqueous media. 1. Preparation and basic characterization. Langmuir 2001, 17 (14), 4240-4244 DOI: 10.1021/la010246x.

51. Tsitsilianis, C.; Voulgaris, D.; Stepanek, M.; Podhajecka, K.; Prochazka, K.; Tuzar, Z.; Brown, W. Polystyrene/poly(2-vinylpyridine) heteroarm star copolymer micelles in aqueous media and onion type micelles stabilized by diblock copolymers. Langmuir 2000, 16 (17), 6868-6876 DOI: 10.1021/la000176e.

52. Borovinskii, A. L.; Khokhlov, A. R. Microphase separation in a mixture of block copolymers in the strong segregation regime. Macromolecules 1998, 31 (4), 1180-1187 DOI: $10.1021 / \mathrm{ma} 970622 \mathrm{~d}$.

53. Martin, T. J.; Prochazka, K.; Munk, P.; Webber, S. E. pH-dependent micellization of poly(2-vinylpyridine)-block-poly(ethylene oxide). Macromolecules 1996, 29 (18), 6071-6073 DOI: 10.1021/ma960629f. 
54. Betthausen, E.; Drechsler, M.; Fortsch, M.; Schacher, F. H.; Muller, A. H. E. Dual stimuli-responsive multicompartment micelles from triblock terpolymers with tunable hydrophilicity. Soft Matter 2011, 7 (19), 8880-8891 DOI: 10.1039/c1sm05822c.

55. Schacher, F.; Walther, A.; Muller, A. H. E. Dynamic Multicompartment-Core Micelles in Aqueous Media. Langmuir 2009, 25 (18), 10962-10969 DOI: 10.1021/la901182c.

56. Cai, Y. L.; Armes, S. P. A Zwitterionic ABC triblock copolymer that forms a "Trinity" of micellar aggregates in aqueous solution. Macromolecules 2004, 37 (19), 7116-7122 DOI: $10.1021 / \mathrm{ma} 048789 \mathrm{~b}$.

57. Sfika, V.; Tsitsilianis, C.; Kiriy, A.; Gorodyska, G.; Stamm, M. pH responsive heteroarm starlike micelles from double hydrophilic ABC terpolymer with ampholitic A and C blocks. Macromolecules 2004, 37 (25), 9551-9560 DOI: 10.1021/ma048621q.

58. Laaser, J. E.; Jiang, Y. M.; Petersen, S. R.; Reineke, T. M.; Lodge, T. P. Interpolyelectrolyte Complexes of Polycationic Micelles and Linear Polyanions: Structural Stability and Temporal Evolution. Journal of Physical Chemistry B 2015, 119 (52), 1591915928 DOI: $10.1021 /$ acs.jpcb.5b09010.

59. Matejicek, P.; Uchman, M.; Lokajova, J.; Stepanek, M.; Prochazka, K.; Spirkova, M. Interpolymer complexes based on the core/shell micelles. Interaction of polystyrene-blockpoly(methacrylic acid) micelles with linear poly(2-vinylpyridine) in 1,4-dioxane water mixtures and in aqueous media. Journal of Physical Chemistry B 2007, 111 (29), 8394-8401 DOI: $10.1021 / \mathrm{jp} 0685075$.

60. Chelushkin, P. S.; Lysenko, E. A.; Bronich, T. K.; Eisenberg, A.; Kabanov, A. V.; Kabanov, V. A. Interpolyelectrolyte complexes of a cationic amphiphilic diblock copolymer and an oppositely charged linear polyanion. Polymer Science Series A 2004, 46 (5), 485-490.

61. Pergushov, D. V.; Remizova, E. V.; Gradzielski, M.; Lindner, P.; Feldthusen, J.; Zezin, A. B.; Muller, A. H. E.; Kabanov, V. A. Micelles of polyisobutylene-blockpoly(methacrylic acid) diblock copolymers and their water-soluble interpolyelectrolyte complexes formed with quaternized poly(4-vinylpyridine). Polymer 2004, 45 (2), 367-378 DOI: 10.1016/j.polymer.2003.10.086.

62. Lysenko, E. A.; Bronich, T. K.; Slonkina, E. V.; Eisenberg, A.; Kabanov, V. A.; Kabanov, A. V. Block ionomer complexes with polystyrene core-forming block in selective solvents of various polarities. 1. Solution behavior and self-assembly in aqueous media. Macromolecules 2002, 35 (16), 6351-6361 DOI: 10.1021/ma020048s.

63. Skandalis, A.; Pispas, S. pH- and thermo-responsive solution behavior of amphiphilic, linear triblock terpolymers. Polymer 2018, 157, 9-18 DOI: 10.1016/j.polymer.2018.10.023.

64. Verduzco, R.; Li, X. Y.; Peseka, S. L.; Steinc, G. E. Structure, function, self-assembly, and applications of bottlebrush copolymers. Chemical Society Reviews 2015, 44 (8), 2405 2420 DOI: $10.1039 / \mathrm{c} 4 \mathrm{cs} 00329 \mathrm{~b}$.

65. Riess, G. Micellization of block copolymers. Progress in Polymer Science 2003, 28 (7), 1107-1170 DOI: 10.1016/s0079-6700(03)00015-7. 
66. Uchman, M.; Gradzielski, M.; Angelov, B.; Tosner, Z.; Oh, J.; Chang, T.; Stepanek, M.; Prochazka, K. Thermodynamic and Kinetic Aspects of Coassembly of PEO-PMAA Block Copolymer and DPCl Surfactants into Ordered Nanoparticles in Aqueous Solutions Studied by ITC, NMR, and Time-Resolved SAXS Techniques. Macromolecules 2013, 46 (6), 21722181 DOI: $10.1021 / \mathrm{ma} 302503 \mathrm{w}$.

67. Richardson, J. J.; Bjornmalm, M.; Caruso, F. Technology-driven layer-by-layer assembly of nanofilms. Science 2015, 348 (6233), DOI: 10.1126/science.aaa2491.

68. Hammond, P. T. Building biomedical materials layer-by-layer. Materials Today 2012, 15 (5), 196-206 DOI: 10.1016/s1369-7021(12)70090-1.

69. Ariga, K.; Hill, J. P.; Ji, Q. M. Layer-by-layer assembly as a versatile bottom-up nanofabrication technique for exploratory research and realistic application. Physical Chemistry Chemical Physics 2007, 9 (19), 2319-2340 DOI: 10.1039/b700410a.

70. Schneider, G.; Decher, G. From functional core/shell nanoparticles prepared via layerby-layer deposition to empty nanospheres. Nano Letters 2004, 4 (10), 1833-1839 DOI: $10.1021 / \mathrm{n} 10490826$.

71. Delisavva, F.; Uchman, M.; Skvarla, J.; Wozniak, E.; Pavlova, E.; Slouf, M.; Garamus, V. M.; Prochazka, K.; Stepanek, M. Influence of Corona Structure on Binding of an Ionic Surfactant in Oppositely Charged Amphiphilic Polyelectrolyte Micelles. Langmuir 2016, 32 (16), 4059-4065 DOI: 10.1021/acs.langmuir.6b00700.

72. Coelho, J. F. J.; Carvalho, E. Y.; Marques, D. S.; Popov, A. V.; Goncalves, P. M.; Gil, M. H. Synthesis of poly(lauryl acrylate) by single-electron transfer/degenerative chain transfer living radical polymerization catalyzed by Na2S2O4 in water. Macromolecular Chemistry and Physics 2007, 208 (11), 1218-1227 DOI: 10.1002/macp.200700015.

73. Uchman, M.; Stepanek, M.; Prevost, S.; Angelov, B.; Bednar, J.; Appavou, M. S.; Gradzielski, M.; Prochazka, K. Coassembly of Poly(ethylene oxide)-block-poly(methacrylic acid) and N-Dodecylpyridinium Chloride in Aqueous Solutions Leading to Ordered Micellar Assemblies within Copolymer Aggregates. Macromolecules 2012, 45 (16), 6471-6480 DOI: $10.1021 / \mathrm{ma} 301510 \mathrm{j}$.

74. Havrankova, J.; Limpouchova, Z.; Stepanek, M.; Prochazka, K. Self-assembly of heteroarm star copolymers - A Monte Carlo study. Macromolecular Theory and Simulations 2007, 16 (4), 386-398 DOI: 10.1002/mats.200600086.

75. Jelinek, K.; Uhlik, F.; Limpouchova, Z.; Prochazka, K. Mean-field study of poly(methacrylic acid) shells in partly hydrophobically modified amphiphilic block copolymer micelles in polar solvents. Journal of Physical Chemistry B 2003, 107 (32), 82418247 DOI: $10.1021 /$ jp0224463.

76. Matejicek, P.; Humpolickova, J.; Prochazka, K.; Tuzar, Z.; Spirkova, M.; Hof, M.; Webber, S. E. Hybrid block copolymer micelles with partly hydrophobically modified polyelectrolyte shells in polar and aqueous media: Experimental study using fluorescence correlation spectroscopy, time-resolved fluorescence, light scattering, and atomic force microscopy. Journal of Physical Chemistry B 2003, 107 (32), 8232-8240 DOI: $10.1021 / \mathrm{jp} 022221 \mathrm{~s}$. 
77. Uhlik, F.; Limpouchova, Z.; Jelinek, K.; Prochazka, K. A Monte Carlo study of shells of hydrophobically modified amphiphilic copolymer micelles in polar solvents. Journal of Chemical Physics 2003, 118 (24), 11258-11264 DOI: 10.1063/1.1575732.

78. Matejicek, P.; Uhlik, F.; Limpouchova, Z.; Prochazka, K.; Tuzar, Z.; Webber, S. Experimental study of hydrophobically modified amphiphilic block copolymer micelles using light scattering and nonradiative excitation energy transfer. Macromolecules 2002, 35 (25), 9487-9496 DOI: 10.1021/ma012074g.

79. Uhlik, F.; Limpouchova, Z.; Matejicek, P.; Prochazka, K.; Tuzar, Z.; Webber, S. E. Nonradiative excitation energy transfer in hydrophobically modified amphiphilic block copolymer micelles. theoretical model and Monte Carlo simulations. Macromolecules 2002, 35 (25), 9497-9505 DOI: 10.1021/ma012073o.

80. Viduna, D.; Limpouchova, Z.; Prochazka, K. Conformations of self-avoiding tethered chains and nonradiative energy transfer and migration in dense and constrained systems. A model for cores of polymeric micelles. Macromolecules 1997, 30 (23), 7263-7272 DOI: $10.1021 / \mathrm{ma} 970002 \mathrm{c}$.

81. Limpouchova, Z.; Prochazka, K. A Monte-Carlo study of insoluble block orientations in swollen cores of multimolecular block-copolymer micelles. Collection of Czechoslovak Chemical Communications 1994, 59 (4), 803-819 DOI: 10.1135/cccc19940803.

82. Koppel, D. E. Analysis of macromolecular polydispersity in intensity correlation spectroscopy: the method of cumulants. The Journal of Chemical Physics 1972, 57 (11), 4814-4820.

83. Danino, D. Cryo-TEM of soft molecular assemblies. Current Opinion in Colloid \& Interface Science 2012, 17 (6), 316-329 DOI: https://doi.org/10.1016/j.cocis.2012.10.003.

84. Brandrup, J.; Immergut, E. H.; Grulke, E. A.; Abe, A.; Bloch, D. R., Polymer handbook. Wiley New York: 1999; Vol. 89.

85. Hoogerbrugge, P. J.; Koelman, J. M. V. A. Simulating microscopic hydrodynamic phenomena with dissipative particle dynamics. Europhysics Letters 1992, 19 (3), 155-160 DOI: $10.1209 / 0295-5075 / 19 / 3 / 001$.

86. Groot, R. D.; Rabone, K. L. Mesoscopic simulation of cell membrane damage, morphology change and rupture by nonionic surfactants. Biophysical Journal 2001, 81 (2), 725-736.

87. Groot, R. D.; Warren, P. B. Dissipative particle dynamics: Bridging the gap between atomistic and mesoscopic simulation. Journal of Chemical Physics 1997, 107 (11), 4423-4435 DOI: $10.1063 / 1.474784$.

88. Espanol, P.; Warren, P. Statistical-mechanics of dissipative particle dynamics. Europhysics Letters 1995, 30 (4), 191-196 DOI: 10.1209/0295-5075/30/4/001.

89. Posel, Z.; Svoboda, M.; Limpouchova, Z.; Lisal, M.; Prochazka, K. Adsorption of amphiphilic graft copolymers in solvents selective for the grafts on a lyophobic surface: a coarse-grained simulation study. Physical Chemistry Chemical Physics 2018, 20 (9), 65336547 DOI: $10.1039 / \mathrm{c} 7 \mathrm{cp} 08327 \mathrm{k}$. 
90. Lisal, M.; Sindelka, K.; Sucha, L.; Limpouchova, Z.; Prochazka, K. Dissipative particle dynamics simulations of polyelectrolyte self-assemblies. Methods with explicit electrostatics. Polymer Science Series C 2017, 59 (1), 77-101 DOI:

$10.1134 / \mathrm{s} 1811238217010052$.

91. Posel, Z.; Svoboda, M.; Colina, C. M.; Lisal, M. Flow and aggregation of rod-like proteins in slit and cylindrical pores coated with polymer brushes: an insight from dissipative particle dynamics. Soft Matter 2017, 13 (8), 1634-1645 DOI: 10.1039/C6SM02751B.

92. Teng, C. Y.; Sheng, Y. J.; Tsao, H. K. Surface Segregation and Bulk Aggregation in an Athermal Thin Film of Polymer-Nanoparticle Blends: Strategies of Controlling Phase Behavior. Langmuir 2017, 33 (10), 2639-2645 DOI: 10.1021/acs.langmuir.6b04681.

93. Wang, Z.; Sun, S.; Li, C.; Hu, S.; Faller, R. Controllable multicompartment morphologies from cooperative self-assembly of copolymer-copolymer blends. Soft Matter 2017, 13 (35), 5877-5887 DOI: 10.1039/c7sm01194f.

94. Wang, C. L.; Ma, S. Y.; Hu, Y.; Wang, R. Hierarchical Colloidal Polymeric Structure from Surfactant-Like Amphiphiles in Selective Solvents. Langmuir 2017, 33 (14), 3427-3433 DOI: 10.1021/acs.langmuir.6b04509.

95. Zhang, J.; Kong, W.; Duan, H. Soft Confinement-Induced Morphologies of the Blends of AB Diblock Copolymers and C Homopolymers. Langmuir 2017, 33 (12), 3123-3133 DOI: 10.1021/acs.langmuir.7b00181.

96. Lisal, M.; Limpouchova, Z.; Prochazka, K. The self-assembly of copolymers with one hydrophobic and one polyelectrolyte block in aqueous media: a dissipative particle dynamics study. Physical Chemistry Chemical Physics 2016, 18 (24), 16127-16136 DOI:

10.1039/c6cp00341a.

97. Prochazka, K.; Sindelka, K.; Wang, X.; Limpouchova, Z.; Lisal, M. Self-assembly and co-assembly of block polyelectrolytes in aqueous solutions. Dissipative particle dynamics with explicit electrostatics. Molecular Physics 2016, 114 (21), 3077-3092 DOI:

10.1080/00268976.2016.1225130.

98. Cheng, J. L.; Vishnyakov, A.; Neimark, A. V. Morphological Transformations in Polymer Brushes in Binary Mixtures: DPD Study. Langmuir 2014, 30 (43), 12932-12940 DOI: $10.1021 / 1 \mathrm{a} 503520 \mathrm{e}$.

99. Huang, J.-H.; Ma, Z.-X.; Luo, M.-B. Self-Assembly of Rod-Coil Diblock Copolymers within a Rod-Selective Slit: A Dissipative Particle Dynamics Simulation Study. Langmuir 2014, 30 (21), 6267-6273 DOI: 10.1021/la501023a.

100. Chen, H. Y.; Ruckenstein, E. Formation and Degradation of Multicomponent Multicore Micelles: Insights from Dissipative Particle Dynamics Simulations. Langmuir 2013, 29 (18), 5428-5434 DOI: 10.1021/la400033s.

101. Sheng, Y. P.; Yang, X. P.; Yan, N.; Zhu, Y. T. Janus-like spheres, disks, rings, cylinders, and vesicles from the self-assembly of mixture of $\mathrm{AB}$ and $\mathrm{BC}$ diblock copolymers in A- and C-selective solvents. Soft Matter 2013, 9 (27), 6254-6262 DOI:

10.1039/c3sm00029j. 
102. Baulin, V. A.; Trizac, E. Self-assembly of spherical interpolyelectrolyte complexes from oppositely charged polymers. Soft Matter 2012, 8 (25), 6755-6766 DOI:

$10.1039 / \mathrm{c} 2 \mathrm{sm} 25423 \mathrm{a}$.

103. Chi, P.; Wang, Z.; Li, B. H.; Shi, A. C. Soft Confinement-Induced Morphologies of Diblock Copolymers. Langmuir 2011, 27 (18), 11683-11689 DOI: 10.1021/la202448c.

104. Chennamsetty, N.; Bock, H.; Lisal, M.; Brennan, J. K., An introduction to coarsegraining approaches: linking atomistic and mesoscales. Wiley-VCH: Weinheim: 2010; Vol. 6.

105. Li, Z.; Dormidontova, E. E. Kinetics of Diblock Copolymer Micellization by Dissipative Particle Dynamics. Macromolecules 2010, 43 (7), 3521-3531 DOI:

10.1021/ma902860j.

106. Sindelka, K.; Limpouchova, Z.; Prochazka, K. Computer study of the solubilization of polymer chains in polyelectrolyte complex cores of polymeric nanoparticles in aqueous media. Physical Chemistry Chemical Physics 2018, 20 (47), 29876-29888 DOI:

10.1039/c8cp05907a.

107. Karayianni, M.; Pispas, S., Self-Assembly of Amphiphilic Block Copolymers in Selective Solvents. In Fluorescence Studies of Polymer Containing Systems, Prochazka, K., Ed. Springer: 2016; pp 27-63. 